\title{
OPEN Robotic pancreaticoduodenectomy provides better histopathological outcomes as compared to its open counterpart: a meta-analysis
}

Xiang Da Dong ${ }^{1,3 凶}$, Daniel Moritz Felsenreich ${ }^{2}$, Shekhar Gogna ${ }^{1}$, Aram Rojas ${ }^{1}$, Ethan Zhang ${ }^{1}$ Michael Dong ${ }^{1}$, Asad Azim ${ }^{1} \&$ Mahir Gachabayov ${ }^{1,4}$

The aim of this meta-analysis was to evaluate whether robotic pancreaticoduodenectomy (PD) may provide better clinical and pathologic outcomes compared to its open counterpart. The Pubmed, EMBASE, and Cochrane Library were systematically searched. Overall postoperative morbidity and resection margin involvement rate were the primary endpoints. Secondary endpoints included operating time, estimated blood loss (EBL), incisional surgical site infection (SSI) rate, length of hospital stay (LOS), and number of lymph nodes harvested. Twenty-four studies totaling 12,579 patients $(2,175$ robotic PD and 10,404 open PD were included. Overall postoperative mortality did not significantly differ $[\mathrm{OR}(95 \% \mathrm{Cl})=0.86(0.74,1.01) ; \mathrm{p}=0.06]$. Resection margin involvement rate was significantly lower in robotic PD $[15.6 \%$ vs. $19.9 \%$; OR $(95 \% \mathrm{Cl})=0.64(0.41,1.00) ; \mathrm{p}=0.05$; NNT = 23]. Operating time was significantly longer in robotic PD $[M D(95 \% \mathrm{Cl})=75.17(48.05,102.28)$; $\mathrm{p}<0.00001]$. EBL was significantly decreased in robotic PD [MD $(95 \% \mathrm{Cl})=-191.35(-238.12$, - 144.59); $\mathrm{p}<0.00001]$. Number of lymph nodes harvested was significantly higher in robotic PD $[\mathrm{MD}(95 \% \mathrm{Cl})=2.88(1.12,4.65) ; \mathrm{p}=0.001]$. This meta-analysis found that robotic PD provides better histopathological outcomes as compared to open PD at the cost of longer operating time. Furthermore, robotic PD did not have any detrimental impact on clinical outcomes, with lower wound infection rates.

Pancreatic surgery has made tremendous progress over the last several decades. With the introduction of minimally invasive techniques, adoption of laparoscopy and robotic platforms for performance of complex pancreatic surgery has evolved as well. Pancreaticoduodenectomy (PD) remains one of the most technically challenging surgeries in the current era ${ }^{1-3}$. Prior to the introduction of the robotic platform, attempts to improve surgical outcome with the use of laparoscopy did not gain widespread adoption ${ }^{4,5}$. Introduction of robotic surgery has overcome some of the limitations from laparoscopic approach ${ }^{6}$. Difficulties related to laparoscopic surgery such as the fulcrum effect, fine instrument manipulation, and reversal of instrument tip from surgical hand-motion created challenges for many surgeons ${ }^{4,5}$.

Following the first case of robotic PD reported in 2003 by Giulianotti et al., multiple studies have reported feasibility and safety of robotic PD in the management of pancreatic head malignancies ${ }^{5,7-11}$. Robotic platform allows performance of complex surgical cases by overcoming the limitations associated with laparoscopic surgery. The advantages of robotic surgery includes 7 degrees of freedom, 3D visualization, and fine tremor reduction ${ }^{3}$. Previous reviews and meta-analyses have found perioperative outcomes of robotic PD to be at worst equivalent to those of open $\mathrm{PD}^{12,13}$. The studies to date have focused on clinical short-term outcomes in patients undergoing robotic PD. However, long-term outcomes of robotic surgery for pancreatic cancer is still scarce in the literature due to the limited longitudinal data available.

Based on longitudinal studies, we know that margin negative (R0) resection for pancreatic cancer translates into improved survival ${ }^{14-16}$. In addition, increased number of lymph nodes retrieved during surgery frequently allows accurate staging and is synonymous with the adequacy of surgical resection ${ }^{17}$. There are currently several

${ }^{1}$ Department of Surgery, Westchester Medical Center, New York Medical College, Valhalla, NY, USA. ${ }^{2}$ Division of General Surgery, Department of Surgery, Vienna Medical University, Vienna, Austria. ${ }^{3}$ Taylor Pavilion, Suite D-365, 100 Woods Road, Valhalla, NY 10595, USA. ${ }^{4}$ Taylor Pavilion, Suite D-361, 100 Woods Road, Valhalla, NY 10595, USA.『email:xddong@hotmail.com; gachabayovmahir@gmail.com 
studies that also reports improved resection margin with the use of robotic platforms ${ }^{12}$. Based on the available literature, we attempted to evaluate the role of robotic PD in comparison to open approach with a focus on short-term clinical outcomes as well as histopathological outcomes such as margin status and nodal harvesting ${ }^{14}$.

\section{Materials and methods}

This systematic review was performed according to the Cochrane Handbook for Systematic Reviews of Interventions ${ }^{18}$ and follows the Preferred Reporting Items for Systematic Reviews and Meta-Analyses (PRISMA) and Meta-analysis Of Observational Studies in Epidemiology (MOOSE) guidelines ${ }^{19,20}$. The protocol of this systematic review was developed prospectively and registered in the International prospective register of systematic reviews PROSPERO: CRD42018112039. Given the summary design nature of this study, Institutional Review Board approval and written consents were not required. The literature search, screening of the records, study selection, extraction and analysis of the data, followed by critical appraisal, were performed by two independent researchers (MG and XDD). The research question was formulated within the PICOTS framework as following:

(P) Population: Adults older than 18 years old undergoing pancreaticoduodenectomy.

(I) Intervention: robotic pancreaticoduodenectomy.

(C) Comparator intervention: open pancreaticoduodenectomy.

(O) Outcomes: operating time, estimated blood lost, postoperative complication rate, postoperative pancreatic fistula (POPF) rate, delayed gastric emptying rate, incisional surgical site infection rate, reoperation rate, length of hospital stay, margin involvement rate, and number of lymph nodes harvested.

(T) Time: Short-term.

(S) Setting: Inpatient.

Eligibility criteria, definitions and endpoints. All experimental or observational clinical studies comparing robotic to open PD for benign and/or malignant disease were eligible for inclusion. Non-comparative descriptive studies, studies comparing any of the interventions of interest to a non-relevant intervention such as laparoscopic pancreaticoduodenectomy or robotic tumor resection followed by mini-laparotomy for reconstruction, and review articles were excluded.

Postoperative complications were classified according to Clavien-Dindo classification ${ }^{21}$. Surgical site infections (SSI) were defined according to the Center for Disease Control National Nosocomial Infections Surveillance System ${ }^{22}$.

The primary endpoints of this systematic review were overall postoperative complication and resection margin involvement rates. Secondary endpoints included operating time, estimated blood loss, postoperative complication rate, postoperative pancreatic fistula rate, rate of delayed gastric emptying, surgical site infection rate, reoperation rate, length of hospital stay, and number of lymph nodes harvested.

Search strategy and study selection. The Pubmed, EMBASE, and Cochrane Library were systematically searched using the following MeSH terms: 'pancreatoduodenectomy', 'pancreaticoduodenectomy', 'whipple', and 'robotic' combined with the Boolean operator 'AND' and all synonyms combined with the Boolean operator 'OR'. In addition, clinicaltrials.gov was searched for any ongoing studies. The details of Pubmed search strategy are presented in Supplement 1. Relevant articles were identified, and the results of the search were screened through the title, abstract and/or full text article. The sensitivity of the search strategy was tested by screening the references of included articles for additional publications.

Data extraction and quality assessment. The data from the included articles were collected to predefined Microsoft Excel tables and studies were assessed for validity by three researchers independently (MG, $\mathrm{XDD}$, and DMF). Extracted data items included publication-specific variables (authors and affiliations, journal and year of publication), study-specific variables (study design, study span, sample size, definitions of interventions and endpoints, conclusions, potential biases), and patient-specific variables (baseline characteristics, intraand postoperative outcomes, pathologic outcomes). Quality assessment of each individual study was performed according to Cochrane Handbook for Systematic Reviews of Interventions on the following items: selection, performance, detection, attrition, selective reporting, and other bias risks ${ }^{18}$. In addition, Risk Of Bias In Nonrandomized Studies (ROBINS-I) tool was utilized to evaluate the quality of observational studies on the following biases: confounding, selection, classification of interventions, deviations of intended comparability, and outcomes ${ }^{23}$.

Statistical analysis. Inverse variance method with mean difference (MD) and standard error as the measure of an effect estimate was used for continuous variables, whereas Mantel-Haenszel method with odds ratios and $95 \%$ confidence intervals (OR $(95 \% \mathrm{CI})$ ) was employed for dichotomous variables. In cases when continuous variables were reported in median and interquartile range in the included studies, mean and standard deviation were estimated using Hozo's formula ${ }^{24}$. Statistical heterogeneity among effect estimates was assessed using Cochran $\mathrm{Chi}^{2}$ and $\mathrm{I}^{2}$, and between-study variance was assessed using Tau ${ }^{2}$ statistic when the $\mathrm{I}^{2}$ was $50 \%$ or greater ${ }^{25}$. Random-effects model was utilized for meta-analysis. The results of the meta-analysis were illustrated on forest plots. Ad-hoc meta-regression analysis with Omnibus test was performed to evaluate the impact of potential confounding factors on outcomes. To assess clinical significance of the statistical findings for dichotomous endpoints, relative risk reduction (RRR), absolute risk reduction (ARR) and number needed to treat/ harm (NNT) with 95\%CI were calculated. Clinical significance of the MD was assessed for numeric endpoints. The variability of the effect of intervention over different settings was assessed using $95 \%$ prediction intervals ${ }^{26}$. Visual assessment of funnel plots and Egger's test were utilized to assess for publication bias. A leave-one-out 


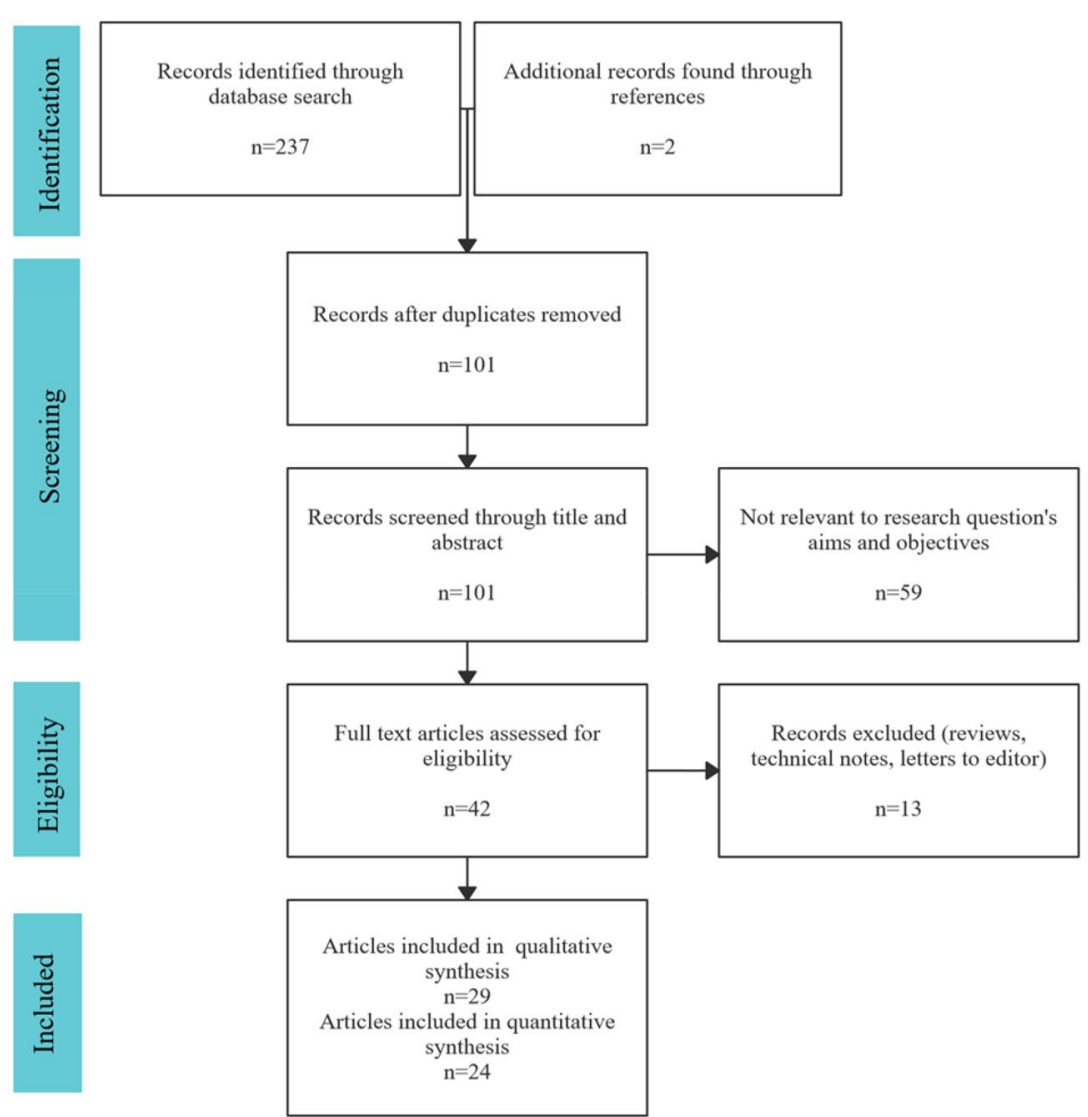

Figure 1. PRISMA flow diagram.

meta-analysis was utilized for sensitivity analysis. A p-value $<0.05$ was considered statistically significant. Certainty of evidence was evaluated using GRADE approach. Statistical analysis was performed using RevMan (version 5.3; Nordic Cochrane Center, Cochrane Collaboration, Copenhagen, Denmark) and CMA Software (Version 3; Biostat, NJ, USA).

\section{Results}

Literature search and study selection. Details of the search strategy and study selection are presented in the PRISMA flowchart (Fig. 1). Four databases were searched and revealed 237 records. Additionally, two articles were found at clinicaltrials.gov and through the references of eligible studies. Twenty-nine studies (including published abstracts of conference proceedings) were included in the qualitative synthesis after excluding duplicates, non-relevant articles, and articles not reporting the outcome of interest.

Quality assessment. The risk of bias summary and graph of the included studies are presented in Fig. 2A,B. The risk of selection, performance and detection bias was high in all included studies given their observational nature. Attrition, reporting, and other bias risks were moderate or low in included studies. The results of quality assessment using the ROBINS-I tool are presented in Supplement 2. Overall risk of bias was assessed as serious in most studies.

Description of included studies. Figure 3 highlights the time span of included studies published from the same institutions, which may increase the risk of duplicate data synthesis. Due to an overlap of the studies by Napoli et al. ${ }^{27}$ and Boogi et al. ${ }^{28}$ from the University of Pisa, only the study by Boogi et al. ${ }^{28}$ was included as it covers a longer time span. An abstract published by Walsh et al. ${ }^{29}$ from Cleveland Clinic was excluded as there was an overlap with the study by Chalikonda et al. $^{30}$ There were five studies from the University of Pittsburgh that overlap to a certain extent. After excluding three (McMillan et al. ${ }^{31}$, Varley et al. ${ }^{32}$ and Wilson et al. ${ }^{33}$ ), studies by Boone et al..$^{34}$ and Cai et al. ${ }^{35}$ with a maximal time span covered and minimal overlap were included.

Twenty-four studies were included in the final quantitative data synthesis, totaling 12,579 patients $(2,175$ robotic PD and 10,404 open PD $)^{8,28,30,34-54}$. Seven studies ${ }^{30,34,36,38,41,50,52}$ were prospective cohort studies and 17 retrospective cohort studies $8,28,35,37,39,40,42-49,51,53,54$. In four of these studies ${ }^{34,40,43,50}$, data were extracted from the abstracts of conference proceedings published in indexed journals. Five studies ${ }^{8,36,38,41,52}$ had the Oxford CEBM 
A

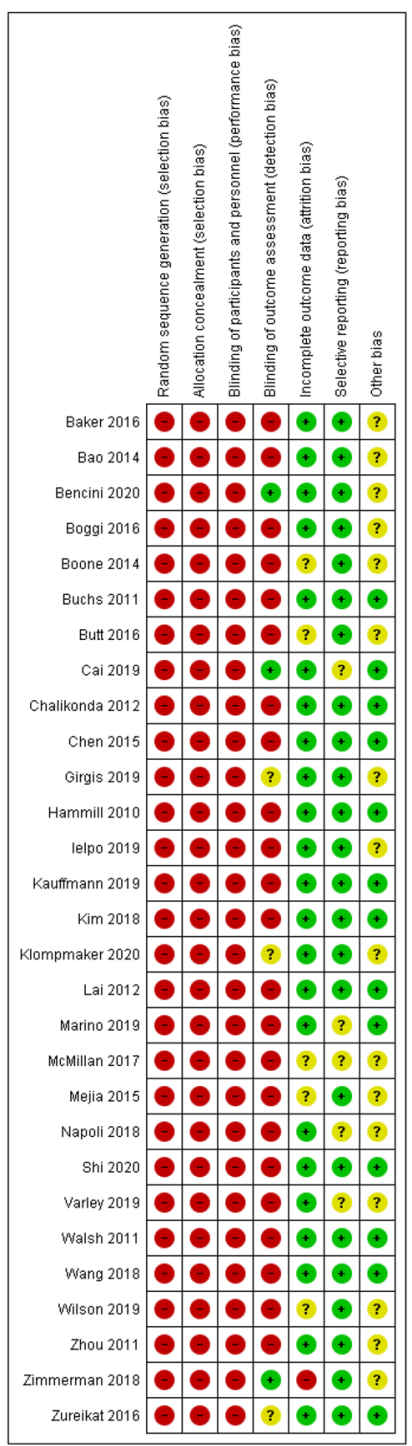

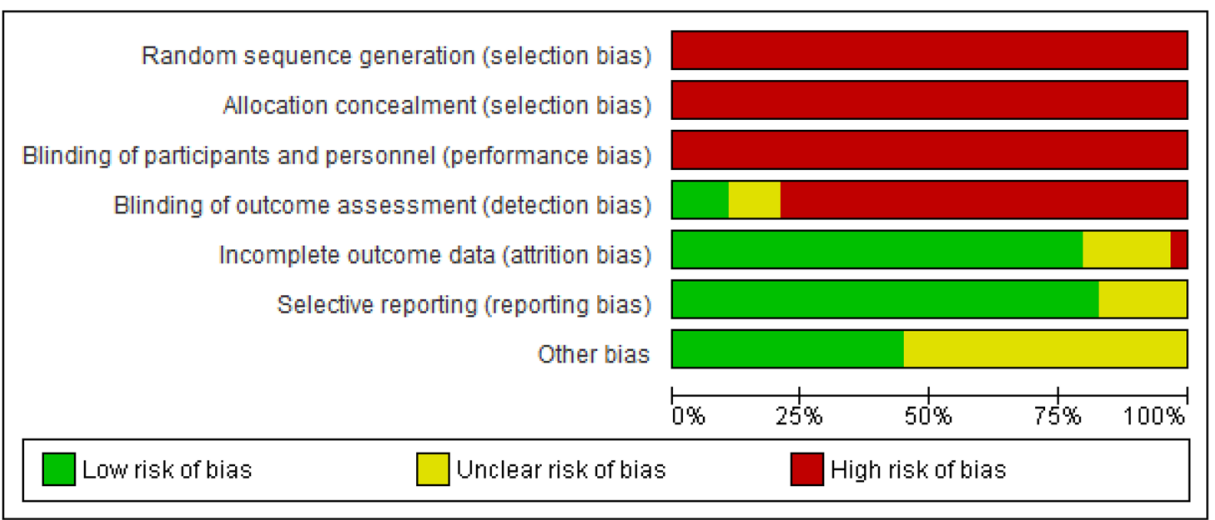

Figure 2. Quality assessment: (A) Risk of bias summary. (B) Risk of bias graph.

level of evidence of $2 \mathrm{~b}$ and 19 studies- $2 \mathrm{c}^{28,30,35,37,39,40,42-51,53-55}$. Three studies ${ }^{8,42,47}$ were multicenter studies with (5,

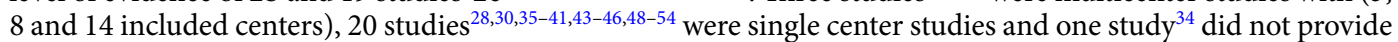
this information. Various primary endpoints were reported in seven studies $35,38,45,47,50,52,54$ and are described in Table 1, whereas 17 studies $^{8,28,30,34,36,37,39-44,46,48,49,51,53}$ did not report/specify this variable.

Description of study populations and interventions. Adult patients from multiple countries (China, Korea, Italy, USA, Russia, Netherlands, Belgium, France, Turkey, Germany, UK and Spain) were involved in the 24 included studies. 17 studies $8,28,30,35-39,41,43,44,46-48,51,52,54$ included patients with benign and malignant diseases, four studies ${ }^{42,45,49,53}$ only malignant diseases and three studies ${ }^{34,40,50}$ did not describe the indications. The primarily involved organs were the pancreas, biliary tract and duodenum in 16 studies $8,28,36-39,41,42,46-49,51-54$; one study ${ }^{43}$ only involved only pancreatic and biliary tract diseases and one study ${ }^{45}$ only pancreatic cancer. Six studies $^{30,34,35,40,44,50}$ did not provide this information (Table 1).

Patients' baseline characteristics are summarized in Table 2. The definitions of the interventions are summarized in Table 3. Robotic PD was a totally robotic procedure in 17 studies $8,28,34-36,38,39,41,42,44,45,47,49,51-54$. A hybrid procedure was performed in four studies $\mathrm{s}^{30,37,46,48}$ and three studies ${ }^{40,43,50}$ did not specify the type of the procedure. Six studies reported DaVinci console type (both $\mathrm{Si} / \mathrm{Xi}$ in three studies ${ }^{38,44,52} ; \mathrm{Si}^{49,50}$ in two and $\mathrm{S}$ in one ${ }^{41}$ ).

Intervention categories that were described for both open and robotic surgery included type of procedure (Whipple procedure, pylorus preserving $\mathrm{PD}$, or multivisceral resection) and type of anastomosis (pancreaticojejunostomy, pancreaticogastrostomy and/or duct-to-mucosa). Vein resection was reported in 10 studies $^{8,28,30,35,36,38,41,42,46,47}$ and was up to $25 \%$ in the robotic and up to $38 \%$ in the open group. Further categories 


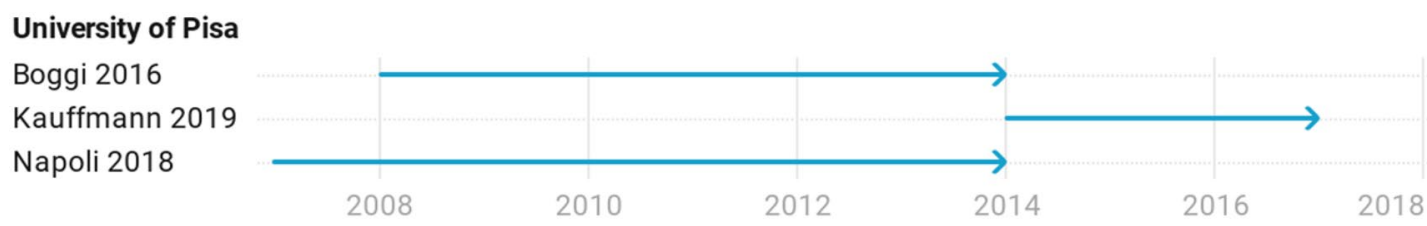

\section{Methodist Dallas Medical Center}

Butt 2016

Mejia 2015
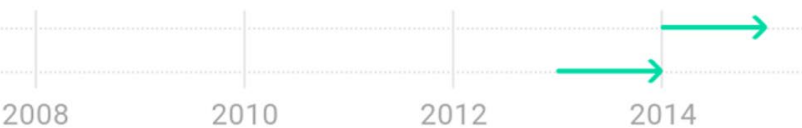

2008 2010

2012

$2014 \quad 2016$

\section{Ruijin Hospital}

Chen 2015

Shi 2020
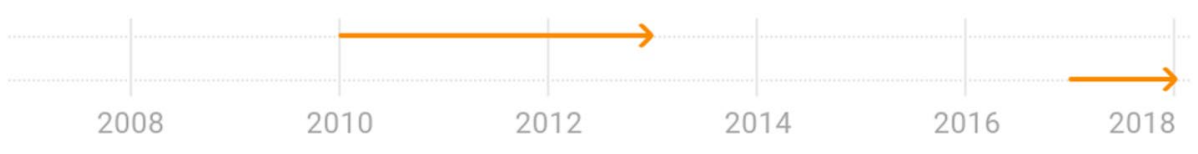

Cleveland Clinic

Chalikonda 2012

Walsh 2011
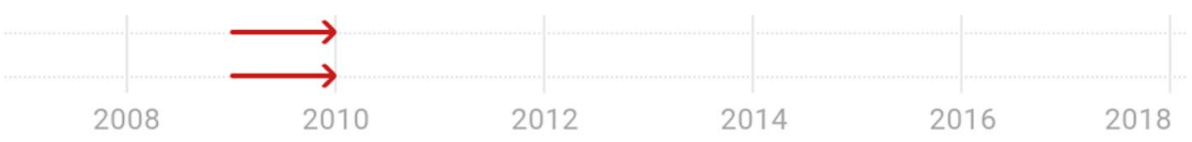

University of Pittsburg

Boone 2014
Cai 2019
McMillan 2017
Varley 2019
Wilson 2019
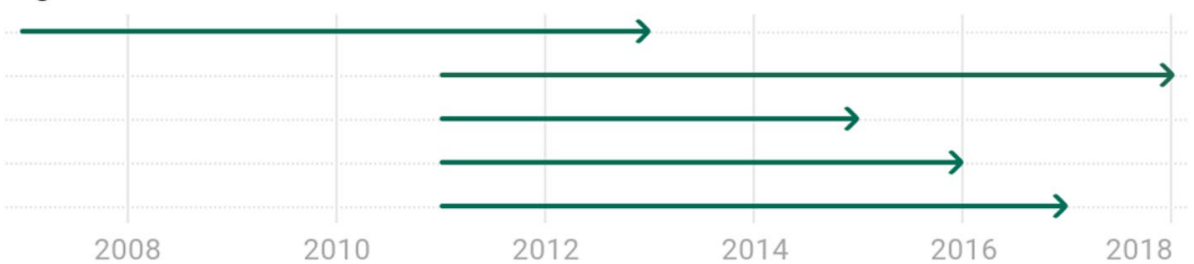

Figure 3. Arrow plot of study spans of included studies with the risk of duplicate data synthesis.

described in Table 3 were routine or selective placement of pancreaticojejunal anastomotic stent, antecolic or retrocolic location of the jejunal loop, and routine or selective use of abdominal drainage.

Meta-analysis. All 24 studies, regardless of the evidence level and risk of bias, were included in this metaanalysis. Primary outcomes were overall postoperative morbidity and margin involvement rate. Secondary clinical outcomes were operating time, estimated blood loss, postoperative pancreatic fistula (POPF), delayed gastric emptying (DGE), surgical site infection (SSI), reoperation rate, and length of hospital stay. An additional secondary outcome was the number of lymph nodes harvested.

Primary endpoints. Overall postoperative morbidity. Overall postoperative morbidity as a clinical primary outcome was reported in 18 studies (1052 robotic PD vs. 8206 open PD). The statistical among-study heterogeneity was low $\left(\mathrm{I}^{2}=0 \%\right)$. The overall postoperative morbidity rate was $42.6 \%(448 / 1,052)$ in robotic PD vs. $54.4 \%(4,464 / 8,206)$ in open PD. This difference was not statistically significant [OR $(95 \% \mathrm{CI})=0.86(0.74,1.01)$; $\mathrm{p}=0.06$ ] (Fig. 4A). The RRR was $22 \%$ and the NNT was $9(7,12)$ (Table 4$) .95 \%$ prediction interval was $0.72,1.02$ with moderate GRADE certainty of evidence (Table 4$)$.

Resection margin involvement rate. Resection margin involvement rate was reported in 17 studies (784 robotic PD vs. 1886 open $\mathrm{PD})$. The statistical among-study heterogeneity was moderate $\left(\mathrm{I}^{2}=56 \%\right.$; $\left.\mathrm{Tau}^{2}=0.40\right)$. The margin involvement rate was $15.6 \%(122 / 784)$ in robotic PD vs. $19.9 \%(376 / 1886)$ in open PD. This difference was statistically and clinically significant $[\mathrm{OR}(95 \% \mathrm{CI})=0.64(0.41,1.00) ; \mathrm{p}=0.05 ; \mathrm{NNT}=23(13,79)]$ (Fig. 4B) (Table 4). 95\% prediction interval was 0.15 , 2.68 with moderate GRADE certainty of evidence (Table 4).

Secondary endpoints. Operating time. Operation time was reported in 23 studies (2,086 robotic PD vs. 10,131 open PD) and was significantly longer in robotic PD $[\mathrm{MD}(95 \% \mathrm{CI})=75.17(48.05,102.28) ; \mathrm{p}<0.00001]$ with high among-study statistical heterogeneity $\left(\mathrm{I}^{2}=99 \%\right.$; $\left.\mathrm{Tau}^{2}=3956.67\right)$ (Fig. $\left.5 \mathrm{~A}\right)$. Although the clinical importance of the MD was assessed to be moderate, $95 \%$ prediction interval was - 58.77, 209.11 and GRADE certainty of evidence was low (Table 4). 


\begin{tabular}{|c|c|c|c|c|c|c|c|c|c|}
\hline Author & Publication & Design & $\begin{array}{l}\text { Number } \\
\text { of centers } \\
\text { involved }\end{array}$ & $\begin{array}{l}\text { Primary } \\
\text { endpoint(s) }\end{array}$ & $\begin{array}{l}\text { Sample } \\
\text { size (total } \\
\mathrm{n}=12,579 \text { ) }\end{array}$ & 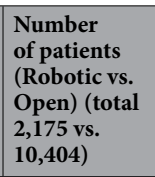 & $\begin{array}{l}\text { Indication for } \\
\text { surgery (benign } \\
\text { or malignant } \\
\text { disease) }\end{array}$ & $\begin{array}{l}\text { Primarily } \\
\text { involved organ } \\
\text { (pancreas, } \\
\text { biliary tract, } \\
\text { duodenum) }\end{array}$ & $\begin{array}{l}\text { Level of } \\
\text { evidence } \\
\text { (Oxford } \\
\text { CEBM) }\end{array}$ \\
\hline Baker & $\begin{array}{l}\text { Int J Med Robot } \\
2016^{36}\end{array}$ & $\begin{array}{l}\text { Prospective } \\
\text { cohort study } \\
(2012-2013)\end{array}$ & 1 & NS & 71 & 22 vs. 49 & $B+M$ & $\mathrm{P}+\mathrm{BT}+\mathrm{D}$ & $2 \mathrm{~b}$ \\
\hline Bao & $\begin{array}{l}\text { J Gastrointest } \\
\text { Surg } 2014^{37}\end{array}$ & \begin{tabular}{|l} 
Retrospective \\
cohort study \\
$(2009-2011)$
\end{tabular} & 1 & NS & 56 & 28 vs. 28 & $B+M$ & $\mathrm{P}+\mathrm{BT}+\mathrm{D}$ & $2 c$ \\
\hline Bencini & $\begin{array}{l}\text { Surg Endosc } \\
2020^{38}\end{array}$ & \begin{tabular}{|l|}
$\begin{array}{l}\text { Prospective } \\
\text { cohort study } \\
(2014-2018)\end{array}$ \\
\end{tabular} & 1 & $\begin{array}{l}\text { Postoperative } \\
\text { (30-day) events }\end{array}$ & 121 & 38 vs. 83 & $B+M$ & $\mathrm{P}+\mathrm{BT}+\mathrm{D}$ & $2 \mathrm{~b}$ \\
\hline Boggi & $\begin{array}{l}\text { World J Surg } \\
2016^{28}\end{array}$ & $\begin{array}{l}\text { Retrospective } \\
\text { cohort study } \\
(2008-2014) \\
\end{array}$ & 1 & NS & 119 & 83 vs. 36 & $B+M$ & $\mathrm{P}+\mathrm{BT}+\mathrm{D}$ & $2 \mathrm{c}$ \\
\hline Boone & НРB $2014^{34}$ & \begin{tabular}{|l} 
Abstract; \\
Prospective \\
cohort study \\
(2008-2013) \\
\end{tabular} & NR & NS & 156 & 58 vs. 98 & NR & NR & $2 c$ \\
\hline Buchs & $\begin{array}{l}\text { World J Surg } \\
2011^{39}\end{array}$ & \begin{tabular}{|l} 
Retrospective \\
cohort study \\
$(2002-2010)$
\end{tabular} & 1 & NS & 83 & 44 vs. 39 & $B+M$ & $\mathrm{P}+\mathrm{BT}+\mathrm{D}$ & $2 c$ \\
\hline Butt & НРВ $2016^{40}$ & \begin{tabular}{|l} 
Abstract; \\
Retrospective \\
cohort study \\
(2014-2015) \\
\end{tabular} & 1 & NS & 67 & 12 vs. 55 & NR & NR & $2 \mathrm{c}$ \\
\hline Cai & $\begin{array}{l}\text { J Gastrointest } \\
\text { Surg } 2019^{35}\end{array}$ & $\begin{array}{l}\text { Retrospective } \\
\text { cohort study } \\
\text { (2011-2018) } \\
\end{array}$ & 1 & CR-POPF rate & 865 & 460 vs. 405 & $B+M$ & NR & $2 \mathrm{c}$ \\
\hline Chalikonda & $\begin{array}{l}\text { Surg Endosc } \\
2012^{30}\end{array}$ & \begin{tabular}{|l|} 
Prospective \\
cohort study \\
$(2009-2010)$ \\
\end{tabular} & 1 & NR & 60 & 30 vs. 30 & $B+M$ & NR & $2 \mathrm{c}$ \\
\hline Chen & $\begin{array}{l}\text { Surg Endosc } \\
2015^{41}\end{array}$ & \begin{tabular}{|l|} 
Prospective \\
cohort study \\
$(2010-2013)$ \\
\end{tabular} & 1 & NR & 180 & 60 vs. 120 & $B+M$ & $\mathrm{P}+\mathrm{BT}+\mathrm{D}$ & $2 \mathrm{~b}$ \\
\hline Girgis & Ann Surg $2019^{42}$ & \begin{tabular}{|l} 
Retrospective \\
cohort study \\
(2011-2016) \\
\end{tabular} & 5 & NR & 361 & 163 vs. 198 & M & $\mathrm{P}+\mathrm{BT}+\mathrm{D}$ & $2 c$ \\
\hline Hammill & HРB $2010^{43}$ & $\begin{array}{l}\text { Abstract; } \\
\text { Retrospective } \\
\text { cohort study } \\
\text { (2005-2009) } \\
\end{array}$ & 1 & NR & 77 & 8 vs. 69 & $B+M$ & $\mathrm{P}+\mathrm{BT}$ & $2 \mathrm{c}$ \\
\hline Ielpo & $\begin{array}{l}\text { Updates Surg } \\
2019^{44}\end{array}$ & \begin{tabular}{|l|} 
Retrospective \\
cohort study \\
$(2010-2017)$ \\
\end{tabular} & 1 & NS & 34 & 17 vs. 17 & $B+M$ & NR & $2 c$ \\
\hline Kauffmann & $\begin{array}{l}\text { Surg Endosc } \\
2019^{45}\end{array}$ & \begin{tabular}{|l|} 
Retrospective \\
cohort study \\
(2014-2017) \\
\end{tabular} & 1 & $\begin{array}{l}\text { Positive margin } \\
\text { rate }\end{array}$ & 268 & 93 vs. 175 & M & $\mathrm{P}$ & $2 \mathrm{c}$ \\
\hline Kim & $\begin{array}{l}\text { J Hepatobiliary } \\
\text { Pancreat Sci } \\
2018^{46} \\
\end{array}$ & \begin{tabular}{|l|} 
Retrospective \\
cohort study \\
$(2015-2017)$ \\
\end{tabular} & 1 & NR & 237 & 51 vs. 186 & $B+M$ & $\mathrm{P}+\mathrm{BT}+\mathrm{D}$ & $2 \mathrm{c}$ \\
\hline Klompmaker & Ann Surg $2020^{47}$ & $\begin{array}{l}\text { Retrospective } \\
\text { cohort study } \\
(2012-2017) \\
\end{array}$ & 14 & $\begin{array}{l}\text { 30-day morbid- } \\
\text { ity }\end{array}$ & 920 & 191 vs. 729 & $B+M$ & $\mathrm{P}+\mathrm{BT}+\mathrm{D}$ & $2 c$ \\
\hline Lai & Int J Surg $2012^{48}$ & \begin{tabular}{|l} 
Retrospective \\
cohort study \\
$(2000-2012)$
\end{tabular} & 1 & NS & 87 & 20 vs. 67 & $B+M$ & $\mathrm{P}+\mathrm{BT}+\mathrm{D}$ & $2 c$ \\
\hline Marino & $\begin{array}{l}\text { J Robot Surg } \\
2019^{49}\end{array}$ & $\begin{array}{l}\text { Retrospective } \\
\text { cohort study } \\
(2014-2016) \\
\end{array}$ & 1 & NR & 70 & 35 vs. 35 & M & $\mathrm{P}+\mathrm{BT}+\mathrm{D}$ & $2 c$ \\
\hline McMillan & $\begin{array}{l}\text { Jama Surg } \\
2017^{31}\end{array}$ & \begin{tabular}{|l|} 
Retrospective \\
cohort study \\
$(2003-2015)$ \\
\end{tabular} & 16 & POPF rate & 2,846 & 185 vs. 2661 & $B+M$ & $\mathrm{P}+\mathrm{BT}+\mathrm{D}$ & $2 c$ \\
\hline Mejia & $\begin{array}{l}\text { Surg Endosc } \\
2015^{50}\end{array}$ & \begin{tabular}{|l|} 
Abstract; \\
Prospective \\
cohort study \\
(2013-2014) \\
\end{tabular} & 1 & Morbidity & 26 & 14 vs. 12 & NR & NR & $2 c$ \\
\hline Napoli $^{\varsigma}$ & $\begin{array}{l}\text { Surg Endosc } \\
2018^{27}\end{array}$ & \begin{tabular}{|l|} 
Retrospective \\
cohort study \\
(2007-2014)
\end{tabular} & 1 & CR-POPF rate & 309 & 82 vs. 227 & $B+M$ & $\mathrm{P}+\mathrm{BT}+\mathrm{D}$ & $2 \mathrm{c}$ \\
\hline Shi & $\begin{array}{l}\text { JAMA Surg } \\
2020^{51}\end{array}$ & \begin{tabular}{|l|} 
Retrospective \\
cohort study \\
(2017-2018) \\
\end{tabular} & 1 & NR & 834 & 200 vs. 634 & $B+M$ & $\mathrm{P}+\mathrm{BT}+\mathrm{D}$ & $2 \mathrm{c}$ \\
\hline \multicolumn{10}{|l|}{ Continued } \\
\hline
\end{tabular}




\begin{tabular}{|c|c|c|c|c|c|c|c|c|c|}
\hline Author & Publication & Design & $\begin{array}{l}\text { Number } \\
\text { of centers } \\
\text { involved }\end{array}$ & $\begin{array}{l}\text { Primary } \\
\text { endpoint(s) }\end{array}$ & $\begin{array}{l}\text { Sample } \\
\text { size (total } \\
\mathrm{n}=12,579)\end{array}$ & \begin{tabular}{|l|}
$\begin{array}{l}\text { Number } \\
\text { of patients } \\
\text { (Robotic vs. }\end{array}$ \\
Open) (total \\
2,175 vs. \\
$10,404)$
\end{tabular} & $\begin{array}{l}\text { Indication for } \\
\text { surgery (benign } \\
\text { or malignant } \\
\text { disease) }\end{array}$ & $\begin{array}{l}\text { Primarily } \\
\text { involved organ } \\
\text { (pancreas, } \\
\text { biliary tract, } \\
\text { duodenum) }\end{array}$ & $\begin{array}{l}\text { Level of } \\
\text { evidence } \\
\text { (Oxford } \\
\text { CEBM) }\end{array}$ \\
\hline Varley ${ }^{\S}$ & НРВ $2019^{32}$ & $\begin{array}{l}\text { Retrospective } \\
\text { cohort study } \\
(2011-2016) \\
\end{array}$ & 1 & $\begin{array}{l}\text { Length of hospi- } \\
\text { tal stay }\end{array}$ & 282 & 133 vs. 149 & $\mathrm{~B}+\mathrm{M}$ & $\mathrm{P}+\mathrm{BT}+\mathrm{D}$ & $2 c$ \\
\hline Walsh $^{\S}$ & $\begin{array}{l}\text { Surg Endosc } \\
2011^{29}\end{array}$ & $\begin{array}{l}\text { Abstract; } \\
\text { Retrospective } \\
\text { cohort study } \\
(2009-2010)\end{array}$ & 1 & NR & 50 & 25 vs. 25 & NR & NR & $2 c$ \\
\hline Wang & Surgery $2018^{52}$ & $\begin{array}{l}\text { Prospective } \\
\text { cohort study } \\
(2012-2017)\end{array}$ & 1 & CR-POPF rate & 296 & 118 vs. 178 & $\mathrm{~B}+\mathrm{M}$ & $\mathrm{P}+\mathrm{BT}+\mathrm{D}$ & $2 \mathrm{~b}$ \\
\hline Wilson ${ }^{\S}$ & HРВ $2019^{33}$ & $\begin{array}{l}\text { Abstract; } \\
\text { Retrospective } \\
\text { cohort study } \\
(2011-2017)\end{array}$ & 1 & NR & 190 & 116 vs. 74 & $\mathrm{M}$ & $\mathrm{P}+\mathrm{BT}+\mathrm{D}$ & $2 c$ \\
\hline Zhou & $\begin{array}{l}\text { Int J Med Robot } \\
2011^{53}\end{array}$ & $\begin{array}{l}\text { Retrospective } \\
\text { cohort study } \\
(2009)\end{array}$ & 1 & NR & 16 & 8 vs. 8 & M & $\mathrm{P}+\mathrm{BT}+\mathrm{D}$ & $2 c$ \\
\hline Zimmerman & HРB $2018^{54}$ & $\begin{array}{l}\text { Retrospective } \\
\text { cohort study } \\
(2014-2015)\end{array}$ & 1 & $\begin{array}{l}\text { 30-day mortality } \\
\text { and morbidity }\end{array}$ & 6547 & 211 vs. 6,336 & $\mathrm{~B}+\mathrm{M}$ & $\mathrm{P}+\mathrm{BT}+\mathrm{D}$ & $2 c$ \\
\hline Zureikat & Ann Surg $2016^{8}$ & $\begin{array}{l}\text { Retrospective } \\
\text { cohort study } \\
(2011-2015)\end{array}$ & 8 & NS & 1028 & 211 vs. 817 & $\mathrm{~B}+\mathrm{M}$ & $\mathrm{P}+\mathrm{BT}+\mathrm{D}$ & $2 b$ \\
\hline
\end{tabular}

Table 1. Characteristics of included studies. $C E B M$ Centers for Evidence-Based Medicine; $B$ benign; $M$ malignant; $P$ pancreas; $B T$ biliary tract; $D$ duodenum; $N R$ not reported; $N S$ not specified. ${ }^{\circledR}$ Studies that were excluded from the quantitative synthesis.

Estimated blood loss. Estimated blood loss was reported in 18 studies (1,549 robotic PD vs. 2,935 open PD) and was significantly lower in robotic PD $[\mathrm{MD}(95 \% \mathrm{CI})=-191.35(-238.12,-144.59) ; \mathrm{p}<0.00001]$ with high among-study statistical heterogeneity $\left(\mathrm{I}^{2}=96 \%\right.$; Tau $\left.^{2}=7,522.30\right)$ (Fig. 5B). Clinical importance of the MD was assessed to be low and 95\% prediction interval was $-382.04,-0.66$ with moderate GRADE certainty of evidence (Table 4).

Postoperative pancreatic fistula rate. POPF rate was reported in 20 studies and did not differ significantly between the two approaches $(18.9 \%(265 / 1,909)$ in robotic PD vs. $16.0 \%(1,589 / 9,921)$ in open PD) [OR $(95 \% \mathrm{CI})=0.89(0.65,1.22) ; \mathrm{p}=0.48 ; \mathrm{NNT}=47(26,267)]$ with high among-study heterogeneity $\left(\mathrm{I}^{2}=64 \%\right.$; $\mathrm{Tau}^{2}=0.26$ ) (Fig. 5C) (Table 4). 95\% prediction interval was $0.29,2.74$ with low GRADE certainty of evidence (Table 4).

Delayed gastric emptying rate. Sixteen studies reported the rate of delayed gastric emptying, which did not differ significantly between the two approaches $(14.7 \%(177 / 1,202)$ in robotic PD vs. $15.2 \%(1,317 / 8,663)$ in open PD) [OR $(95 \% \mathrm{CI})=0.98(0.74,1.30) ; \mathrm{p}=0.87$, with low among-study heterogeneity $\left(\mathrm{I}^{2}=24 \%\right.$; $\left.\mathrm{Tau}^{2}=0.07\right)$ (Fig. 5D). RRR was $3 \%$, NNT was $210,95 \%$ prediction interval was $0.51,1.87$ with very low GRADE certainty of evidence (Table 4).

Incisional surgical site infection rate. Incisional SSI rate was reported in 13 studies and favored robotic PD $(7.9 \%(80 / 1,010)$ in robotic PD vs. $10.1 \%(820 / 8,100)$ in open PD) $[\mathrm{OR}(95 \% \mathrm{CI})=0.45(0.27,74) ; \mathrm{p}=0.002$; $\mathrm{NNT}=46(25,243)]$ with moderate among-study heterogeneity $\left(\mathrm{I}^{2}=52 \%\right.$; Tau $\left.^{2}=0.30\right)$ (Fig. 5E) (Table 4). 95\% prediction interval was $0.12,1.70$ with low GRADE certainty of evidence (Table 4 ).

Reoperation rate. Sixteen studies reported the rate of reoperations (1,521 robotic PD vs. 8,732 open PD), which did not differ significantly between the two approaches $(6.6 \%(100 / 1,521)$ in robotic PD vs. $6.6 \%(577 / 8,732)$ in open $\mathrm{PD})[\mathrm{OR}(95 \% \mathrm{CI})=0.80(0.62,102) ; \mathrm{p}=0.72]$, with low among-study heterogeneity $\left(\mathrm{I}^{2}=0 \%\right.$; $\left.\mathrm{Tau}^{2}=0.00\right)$ (Fig. 5F). RRR was $1 \%$, NNT was $3,007,95 \%$ prediction interval was $0.61,1.04$ with very low GRADE certainty of evidence (Table 4$)$.

Length of hospital stay. Length of hospital stay was reported in 20 studies (1,893 robotic PD vs. 9,903 open PD) and did not differ significantly between the two approaches [MD $(95 \% \mathrm{CI})=-1.00(-1.88,-0.12) ;(\mathrm{p}=0.06)]$ with high among-study heterogeneity $\left(\mathrm{I}^{2}=97 \%\right.$; Tau $\left.^{2}=2.29\right)$ (Fig. $\left.5 \mathrm{G}\right)$. Although the clinical importance of the MD was assessed to be moderate, $95 \%$ prediction interval was $-4.32,2.32$ and GRADE certainty of evidence was very low (Table 4 ). 


\begin{tabular}{|c|c|c|c|c|c|c|c|c|}
\hline \multirow[b]{2}{*}{ Included studies } & \multicolumn{2}{|l|}{ Age (years) } & \multicolumn{2}{|c|}{ Gender ( $\%$ male) } & \multicolumn{2}{|l|}{ BMI $\left(\mathbf{k g} / \mathbf{m}^{2}\right)$} & \multicolumn{2}{|l|}{ ASA $>2$} \\
\hline & Robotic & Open & Robotic & Open & Robotic & Open & Robotic & Open \\
\hline Baker $2016^{36}$ & $63(38-82)^{*}$ & $63(26-86)^{*}$ & $5 \%$ & $63 \%$ & $26(18-35)^{*}$ & $27(16-38)^{*}$ & $68 \%$ & $82 \%$ \\
\hline Bao $2014^{37}$ & $68 \pm 11.2$ & $67.7 \pm 12.5$ & $46 \%$ & $46 \%$ & $26(19-40)^{*}$ & $26(19-40)^{*}$ & NR & NR \\
\hline Bencini $2020^{38}$ & $60(42-73)^{*}$ & $74(56-91)^{*}$ & $58 \%$ & $53 \%$ & $26(18-32)^{*}$ & $24(14-38)^{*}$ & $16 \%$ & $36 \%$ \\
\hline Boggi $2016^{28}$ & $62(50-71)^{*}$ & $64(56-74)^{*}$ & $45 \%$ & $53 \%$ & $24(23-24)^{*}$ & $23(22-25)^{*}$ & $33 \%$ & $36 \%$ \\
\hline Boone $2014^{34}$ & NR & NR & NR & NR & NR & NR & NR & NR \\
\hline Buchs $2011^{39}$ & $63 \pm 14.5$ & $56 \pm 15.8$ & $50 \%$ & $36 \%$ & $27.7 \pm 5.4$ & $24.8 \pm 4.7$ & NR & NR \\
\hline Butt $2016^{40}$ & $\mathrm{NR}$ & $\mathrm{NR}$ & NR & NR & NR & NR & NR & NR \\
\hline Cai $2019^{35}$ & $66.5 \pm 11.0$ & $67.5 \pm 10.7$ & $55 \%$ & $52 \%$ & $27.8 \pm 5.8$ & $27.2 \pm 5.9$ & NR & NR \\
\hline Chalikonda $2012^{30}$ & 62 & 61 & $54 \%$ & $54 \%$ & 24.8 & 25.6 & $53 \%$ & $76 \%$ \\
\hline Chen $2015^{41}$ & $53.6 \pm 13.5$ & $53.8 \pm 14.3$ & $57 \%$ & $54 \%$ & $23.2 \pm 2.7$ & $22.6 \pm 3.4$ & $1.7 \%$ & $1.6 \%$ \\
\hline Girgis $2019^{42}$ & $66.6 \pm 10.9$ & $67.6 \pm 10.3$ & $53 \%$ & $53 \%$ & $27.1 \pm 5.6$ & $26.4 \pm 5.3$ & NR & NR \\
\hline Hammill $2010^{43}$ & 55 & 62.5 & NR & NR & 26.1 & 26.6 & NR & NR \\
\hline Ielpo $2019^{44}$ & $66.8 \pm 9.5$ & $61.4 \pm 11.9$ & $47 \%$ & $59 \%$ & $23.8 \pm 4.1$ & $24.6 \pm 3.36$ & $35 \%$ & $24 \%$ \\
\hline Kauffmann $2019^{45}$ & $65(59-75)^{\star}$ & $73(60-79)^{*}$ & $50 \%$ & $54 \%$ & $23.1 \pm 3.2$ & $24.1 \pm 3.1$ & NR & $\mathrm{NR}$ \\
\hline Kim $2018^{46}$ & $60.7 \pm 11.9$ & $65.4 \pm 10.1$ & $58 \%$ & $47 \%$ & $22.7 \pm 2.5$ & $24.0 \pm 3.1$ & $2 \%$ & $5 \%$ \\
\hline Klompmaker $2020^{47}$ & $\mathrm{NR}$ & $34.6 \pm 11.7$ & NR & $50 \%$ & $\mathrm{NR}$ & $24.8 \pm 4.0$ & NR & $20 \%$ \\
\hline Lai $2012^{48}$ & $66.4 \pm 11.9$ & $62.1 \pm 11.2$ & $60 \%$ & $57 \%$ & $\mathrm{NR}$ & NR & $0 \%$ & $0 \%$ \\
\hline Marino $2019^{49}$ & $60.4(43-72)^{*}$ & $62.3(45-73)^{*}$ & $54 \%$ & $43 \%$ & $23.8(19.4-30.9)^{*}$ & $23.5(18.8-28.1)^{\star}$ & $20 \%$ & $23 \%$ \\
\hline McMillan $2017^{31 ~}$ & $\mathrm{NR}$ & $\mathrm{NR}$ & NR & NR & NR & NR & NR & NR \\
\hline Mejia $2015^{50}$ & $67.3 \pm 8$ & $62 \pm 10$ & $71 \%$ & $58 \%$ & $27 \pm 5$ & $27.2 \pm 5$ & $\mathrm{NR}$ & $\mathrm{NR}$ \\
\hline Napoli $2018^{27 \S}$ & $62(52-71)^{*}$ & $67(60-75)^{*}$ & $44 \%$ & $55 \%$ & $23.5 \pm 0.4$ & $24.8 \pm 0.2$ & $42 \%$ & $66 \%$ \\
\hline Shi $2020^{51}$ & $59.4 \pm 12.6$ & $62.7 \pm 10.5$ & $56 \%$ & $60 \%$ & NR & NR & $4 \%$ & $6 \%$ \\
\hline Varley $2019^{32 \S}$ & $66.3 \pm 10.6$ & $67.0 \pm 10.5$ & $48 \%$ & $53 \%$ & $27.5 \pm 6.1$ & $26.7 \pm 5.6$ & $89 \%$ & $86 \%$ \\
\hline Walsh $2011^{29 \varsigma}$ & 63 & 62 & NR & NR & 24 & 26 & $50 \%$ & $69 \%$ \\
\hline Wang $2018^{52}$ & NR & NR & $50 \%$ & $57 \%$ & NR & NR & NR & NR \\
\hline Wilson $2019^{33 \S}$ & $67.3 \pm 10.3$ & $69.8 \pm 10.2$ & NR & NR & NR & NR & NR & NR \\
\hline Zhou $2011^{53}$ & $64.4 \pm 9.1$ & $59.4 \pm 9.4$ & $63 \%$ & $50 \%$ & NR & NR & NR & NR \\
\hline Zimmerman $2018^{54}$ & $66(68-72)^{*}$ & $65(57-72)^{*}$ & $52 \%$ & $54 \%$ & $27.3(23.8-30.9)^{*}$ & $26.5(23.2-30.2)^{*}$ & NR & NR \\
\hline Zureikat $2016^{8}$ & $67(15-86)^{*}$ & $65(15-93)^{*}$ & $55 \%$ & $52 \%$ & $27.5(18.1-47.6)^{\star}$ & $26.1(14.7-85.5)^{*}$ & NR & NR \\
\hline
\end{tabular}

Table 2. Comparison of patients' baseline characteristics in robotic vs. open pancreaticoduodenectomy. $B M I$ body mass index; ASA American Society of Anesthesiologists; NR not reported. ${ }^{\star}$ Expressed in median and interquartile range. ${ }^{\S}$ Studies that were excluded from the quantitative synthesis.

Number of lymph nodes harvested. Number of lymph nodes harvested was reported in 15 studies (908 robotic $\mathrm{PD}$ vs. 1,953 open $\mathrm{PD})$. Statistical among-study heterogeneity was high $\left(\mathrm{I}^{2}=83 \%\right.$; $\left.\mathrm{Tau}^{2}=9.24\right)$. The difference was statistically significant $[\mathrm{MD}(95 \% \mathrm{CI})=2.88(1.12,4.65) ; \mathrm{p}=0.001]$ (Fig. $5 \mathrm{H})$. Although the clinical importance of the MD was be moderate, $95 \%$ prediction interval was $-3.97,9.73$ and GRADE certainty of evidence was low (Table 4).

Meta-regression analysis. Ad-hoc meta-regression analysis was performed to assess the impact of potential covariates on the statistical findings. Covariates utilized for meta-regression analysis included the central tendency values for age and BMI, proportion of males, proportion patients with ASA $>2$, and study design. A statistically significant correlation was found between overall postoperative mortality and average age in robotic PD (Omnibus $\mathrm{p}=0.040)$ (Fig. 6A). However, only a statistical trend in correlation was found between overall postoperative mortality and open PD (Omnibus $\mathrm{p}=0.075)$ (Fig. 6B). No statistically significant impact of the above-mentioned covariates on margin involvement rate and secondary endpoints was found.

Publication bias and sensitivity analysis. Publication bias was evaluated by visual assessment of symmetry on the funnel plot (Fig. 7) and using Egger's test (Overall postoperative morbidity: $t=0.534, p=0.522$; Margin involvement rate: $\mathrm{t}=0.478, \mathrm{p}=0.641$ ). No significant risk of publication bias was found. A sensitivity analysis of the included observational studies was performed using leave-one-out forest plots. Consecutive exclusion of studies did not significantly impact the findings (Fig. 8). The results of the evaluation of the certainty of evidence are summarized in Table 4. 


\begin{tabular}{|c|c|c|c|c|c|c|c|c|c|c|c|c|c|c|}
\hline \multirow[b]{2}{*}{ Studies } & \multicolumn{8}{|l|}{ Robotic } & \multicolumn{6}{|l|}{ Open } \\
\hline & $\begin{array}{l}\text { Technique } \\
\text { (TR/H) }\end{array}$ & $\begin{array}{l}\text { Console } \\
\text { type }(\mathrm{S} / \mathrm{Si} / \\
\mathrm{Xi})\end{array}$ & $\begin{array}{l}\text { Type of } \\
\text { procedure } \\
\text { (WP/PP/ } \\
\text { MVR) }\end{array}$ & $\begin{array}{l}\text { Vein } \\
\text { resection } \\
(\%)\end{array}$ & $\begin{array}{l}\text { Type of } \\
\text { anastomosis } \\
\text { (PJ/PG/DtM) }\end{array}$ & $\begin{array}{l}\text { PJAS } \\
\text { used } \\
(\mathrm{R} / \mathrm{S})\end{array}$ & $\begin{array}{l}\text { Location } \\
\text { of the } \\
\text { jejunal } \\
\text { loop (AC/ } \\
\text { RC) }\end{array}$ & $\begin{array}{l}\text { Peritoneal } \\
\text { drain used } \\
(\mathrm{R} / \mathrm{S})\end{array}$ & $\begin{array}{l}\text { Type of } \\
\text { procedure } \\
\text { (WP/PP/ } \\
\text { MVR) }\end{array}$ & $\begin{array}{l}\text { Vein } \\
\text { resection } \\
(\%)\end{array}$ & $\begin{array}{l}\text { Type of } \\
\text { anastomosis } \\
\text { (PJ/PG/DtM) }\end{array}$ & $\begin{array}{l}\text { PJAS } \\
\text { used } \\
(\mathbf{R} / \mathbf{S})\end{array}$ & $\begin{array}{l}\text { Location } \\
\text { of the } \\
\text { jejunal } \\
\text { loop (AC/ } \\
\text { RC) }\end{array}$ & $\begin{array}{l}\text { Peritoneal } \\
\text { drain used } \\
\text { (R/S) }\end{array}$ \\
\hline $\begin{array}{l}\text { Baker } \\
2016^{36}\end{array}$ & TR & NR & PP-100\% & $14 \%$ & $\mathrm{PJ}+\mathrm{DtM}$ & NR & $\mathrm{RC}$ & $\mathrm{R}$ & $\begin{array}{l}\text { WP-12\%; } \\
\text { PP- } 88 \%\end{array}$ & $14 \%$ & $\mathrm{PJ}+\mathrm{DtM}$ & NR & $\mathrm{AC}$ & $\mathrm{R}$ \\
\hline $\begin{array}{l}\text { Bao } \\
2014^{37}\end{array}$ & $\mathrm{H}$ & NR & WP \& PP & NR & $\mathrm{PJ} / \mathrm{PG}+\mathrm{DtM}$ & S & $\mathrm{AC} / \mathrm{RC}$ & $\mathrm{R}$ & WP \& PP & NR & $\mathrm{PJ} / \mathrm{PG}+\mathrm{DtM}$ & S & $\mathrm{AC} / \mathrm{RC}$ & $\mathrm{R}$ \\
\hline $\begin{array}{l}\text { Bencini } \\
2020^{38}\end{array}$ & TR & $\mathrm{Si} / \mathrm{Xi}$ & $\begin{array}{l}\text { WP-55\%; } \\
\text { PP- } 45 \%\end{array}$ & $0 \%$ & $P G+D t M$ & NR & NR & $\mathrm{R}$ & $\begin{array}{l}\text { WP-30\%; } \\
\text { PP-70\%; } \\
\text { MVR-13\% }\end{array}$ & $24 \%$ & $\mathrm{PJ} / \mathrm{PG}+\mathrm{DtM}$ & NR & NR & $\mathrm{R}$ \\
\hline $\begin{array}{l}\text { Boggi } \\
2016^{28}\end{array}$ & TR & NR & WP & $8 \%$ & $\mathrm{PJ}+\mathrm{DtM}$ & NR & NR & $\mathrm{R}$ & WP & $11 \%$ & NR & NR & NR & $\mathrm{R}$ \\
\hline $\begin{array}{l}\text { Boone } \\
2014^{34} \\
\end{array}$ & $\mathrm{TR}$ & NR & NR & NR & NR & NR & NR & NR & NR & NR & NR & NR & NR & NR \\
\hline $\begin{array}{l}\text { Buchs } \\
2011^{39}\end{array}$ & TR & NR & WP \& PP & NR & $\mathrm{PJ} / \mathrm{PG}$ & NR & NR & $\mathrm{R}$ & WP & NR & PJ & NR & NR & $\mathrm{R}$ \\
\hline $\begin{array}{l}\text { Butt } \\
2016^{40}\end{array}$ & NR & NR & NR & NR & NR & NR & NR & NR & NR & NR & NR & NR & NR & NR \\
\hline $\begin{array}{l}\text { Cai } \\
2019^{35}\end{array}$ & TR & NR & WP & $15 \%$ & $\mathrm{PJ}+\mathrm{DtM}$ & S & NR & $\mathrm{R}$ & WP & $23 \%$ & $\mathrm{PJ}+\mathrm{DtM}$ & S & NR & $\mathrm{R}$ \\
\hline $\begin{array}{l}\text { Cha- } \\
\text { likonda } \\
2012^{30}\end{array}$ & $\mathrm{H}$ & NR & PP-100\% & $0 \%$ & $\mathrm{PJ}+\mathrm{DtM}$ & $\mathrm{R}$ & $\mathrm{AC}$ & $\mathrm{R}$ & PP-100\% & $0 \%$ & NR & NR & NR & $\mathrm{R}$ \\
\hline $\begin{array}{l}\text { Chen } \\
2015^{41}\end{array}$ & $\mathrm{TR}$ & S & WP & $5 \%$ & $\mathrm{PJ}+\mathrm{DtM}$ & $\mathrm{R}$ & $\mathrm{RC}$ & $\mathrm{R}$ & WP \& PP & $7 \%$ & $\mathrm{PJ} / \mathrm{PG}+\mathrm{DtM}$ & NR & NR & NR \\
\hline $\begin{array}{l}\text { Girgis } \\
2019^{42}\end{array}$ & $\mathrm{TR}$ & NR & WP & $25 \%$ & NR & NR & NR & NR & WP & $38 \%$ & NR & NR & NR & NR \\
\hline $\begin{array}{l}\text { Hammill } \\
2010^{43}\end{array}$ & NR & NR & NR & NR & NR & NR & NR & NR & NR & NR & NR & NR & NR & NR \\
\hline $\begin{array}{l}\text { Ielpo } \\
2019^{44}\end{array}$ & TR & $\mathrm{Si} / \mathrm{Xi}$ & WP & NR & $\mathrm{PJ}+\mathrm{DtM}$ & NR & NR & NR & WP & NR & $\mathrm{PJ}+\mathrm{DtM}$ & NR & NR & NR \\
\hline $\begin{array}{l}\text { Kauff- } \\
\text { mann } \\
2019^{45}\end{array}$ & $\mathrm{TR}$ & NR & WP & NR & $\mathrm{PJ}+\mathrm{DtM}$ & NR & NR & $\mathrm{R}$ & WP & NR & NR & NR & NR & $\mathrm{R}$ \\
\hline $\begin{array}{l}\text { Kim } \\
2018^{46}\end{array}$ & $\mathrm{H}$ & NR & $\begin{array}{l}\text { WP-4\%; } \\
\text { PP-96\% }\end{array}$ & $0 \%$ & $\mathrm{PJ}+\mathrm{DtM}$ & $\mathrm{R}$ & NR & NR & $\begin{array}{l}\text { WP-26\%; } \\
\text { PP- } 74 \%\end{array}$ & $7 \%$ & $\mathrm{PJ}+\mathrm{DtM}$ & $\mathrm{R}$ & NR & NR \\
\hline $\begin{array}{l}\text { Klomp- } \\
\text { maker } \\
2020^{47}\end{array}$ & $\mathrm{TR}$ & NR & $\begin{array}{l}\text { WP-31\%; } \\
\text { PP-67\%; } \\
\text { MVR-2\% }\end{array}$ & $10 \%$ & NR & NR & NR & NR & $\begin{array}{l}\text { WP-31\%; } \\
\text { PP-69\%; } \\
\text { MVR-3\% }\end{array}$ & $10 \%$ & $\mathrm{PJ} / \mathrm{PG}$ & NR & NR & NR \\
\hline $\begin{array}{l}\text { Lai } \\
2012^{48}\end{array}$ & $\mathrm{H}$ & NR & WP & NR & $\mathrm{PJ}+\mathrm{DtM}$ & $\mathrm{R}$ & NR & $\mathrm{R}$ & $\begin{array}{l}\text { WP-94\%; } \\
\text { PP-6\% }\end{array}$ & NR & NR & NR & NR & NR \\
\hline $\begin{array}{l}\text { Marino } \\
2019^{49}\end{array}$ & $\mathrm{TR}$ & $\mathrm{Si}$ & WP & NR & $\mathrm{PJ}+\mathrm{DtM}$ & $\mathrm{R}$ & NR & $\mathrm{R}$ & NR & NR & NR & NR & NR & NR \\
\hline $\begin{array}{l}\text { McMil- } \\
\text { lan } 2017\end{array}$ & $\mathrm{TR}$ & NR & WP & NR & $\mathrm{PJ}+\mathrm{DtM}$ & S & NR & $\mathrm{R}$ & NR & NR & $\mathrm{PJ} / \mathrm{PG}$ & S & NR & S \\
\hline $\begin{array}{l}\text { Mejia } \\
2015^{50}\end{array}$ & NR & $\mathrm{Si}$ & NR & NR & NR & NR & NR & NR & NR & NR & NR & NR & NR & NR \\
\hline $\begin{array}{l}\text { Napoli } \\
2018^{27 \$}\end{array}$ & $\mathrm{TR}$ & NR & $\begin{array}{l}\text { WP-7\%; } \\
\text { PP-93\% }\end{array}$ & $9 \%$ & $\mathrm{PJ}+\mathrm{DtM}$ & S & NR & $\mathrm{R}$ & $\begin{array}{l}\text { WP-15\%; } \\
\text { PP- } 85 \%\end{array}$ & $34 \%$ & $\mathrm{PJ}+\mathrm{DtM}$ & S & NR & $\mathrm{R}$ \\
\hline $\begin{array}{l}\text { Shi } \\
2020^{51}\end{array}$ & $\mathrm{TR}$ & NR & WP & NR & $\mathrm{PJ}+\mathrm{DtM}$ & $\mathrm{R}$ & RC & $\mathrm{R}$ & WP \& PP & NR & $\mathrm{PJ} / \mathrm{PG}+\mathrm{DtM}$ & NR & NR & NR \\
\hline $\begin{array}{l}\text { Varley } \\
2019^{32 \$}\end{array}$ & TR & NR & NR & NR & NR & NR & NR & NR & NR & NR & NR & NR & NR & NR \\
\hline $\begin{array}{l}\text { Walsh } \\
2011^{299}\end{array}$ & $\mathrm{H}$ & NR & WP & NR & NR & NR & NR & NR & WP & NR & NR & NR & NR & NR \\
\hline $\begin{array}{l}\text { Wang } \\
2018^{52}\end{array}$ & $\mathrm{TR}$ & $\mathrm{Si} / \mathrm{Xi}$ & NR & NR & Blumgart PJ & S & $\mathrm{RC}$ & NR & NR & NR & Blungart PJ & S & $\mathrm{RC}$ & NR \\
\hline $\begin{array}{l}\text { Wilson } \\
2019^{339}\end{array}$ & $\mathrm{TR}$ & NR & NR & NR & NR & NR & NR & NR & NR & NR & NR & NR & NR & NR \\
\hline $\begin{array}{l}\text { Zhou } \\
2011^{53}\end{array}$ & $\mathrm{TR}$ & NR & $\begin{array}{l}\text { WP-63\%; } \\
\text { PP-37\% }\end{array}$ & NR & $\mathrm{PJ}+\mathrm{DtM}$ & NR & NR & NR & NR & NR & $\mathrm{PJ} / \mathrm{PG}+\mathrm{DtM}$ & NR & NR & NR \\
\hline $\begin{array}{l}\text { Zim- } \\
\text { merman } \\
2018^{54}\end{array}$ & $\mathrm{TR}$ & NR & NR & NR & NR & NR & NR & NR & NR & NR & NR & NR & NR & NR \\
\hline $\begin{array}{l}\text { Zureikat } \\
2016^{8}\end{array}$ & $\mathrm{TR}$ & NR & $\begin{array}{l}\text { WP-67\%; } \\
\text { PP-33\% }\end{array}$ & $0 \%$ & NR & $\mathrm{S}$ & NR & $\mathrm{R}$ & $\begin{array}{l}\text { WP-54\%; } \\
\text { PP-46\% }\end{array}$ & $0 \%$ & NR & S & NR & S \\
\hline
\end{tabular}

Table 3. Definition of interventions in included studies. TR totally robotic; $H$ hybrid; $W P$ Whipple procedure; $P P$ pylorus preserving; $M V R$ multivisceral resection; $P J$ pancreaticojejunostomy; $P G$ pancreaticogastrostomy; $D t M$ duct-to-mucosa; PJAS pancreatojejunal anastomotic stent; $R$ routinely; $S$ selectively; $A C$ antecolic; $R C$ retrocolic; $N R$ not reported. ${ }^{\S}$ Studies that were excluded from the quantitative synthesis. 
A

Robotic Open Odds Ratio

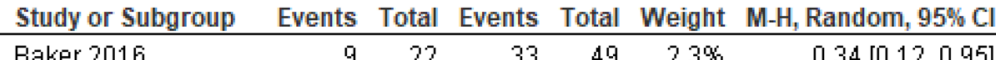

Bencini 2020

Boggi 2016

Boone 2014

Buchs 2011

Chalikonda 2012

Chen 2015

Hammill 2010

lelpo 2019

Kauffmann 2019

Kim 2018

Lai 2012

Marino 2019

Mejia 2015

Wang 2018

Zhou 2011

Zimmerman 2018

Zureikat 2016

Total $(95 \% \mathrm{Cl})$

Total events

$20 \quad 38$

$43 \quad 83 \quad 4.2 \%$

$1.03[0.48,2.23]$

$0.79[0.31,2.00]$

$\begin{array}{lllll}14 & 58 & 24 & 98 & 4.3 \%\end{array}$

$\begin{array}{lllll}16 & 44 & 19 & 39 & 3.2 \%\end{array}$

930

$26 \quad 60$

$13 \quad 30 \quad 2.2 \%$

$0.98[0.46,2.09]$

$0.60[0.25,1.45]$

$0.56[0.19,1.62]$

$0.57[0.30,1.06]$

$1.10[0.20,6.01]$

$0.78[0.19,3.12]$

$0.32[0.07,1.41]$

$0.70[0.30,1.61]$

$1.03[0.38,2.80]$

$0.49[0.18,1.29]$

$0.80[0.15,4.24]$

$0.84[0.53,1.35]$

$0.11[0.01,1.07]$

$1.09[0.82,1.44]$

$0.99[0.69,1.41]$

$0.86[0.74,1.01]$

Heterogeneity: $\mathrm{Tau}^{2}=0.00 ; \mathrm{Ch}^{2}=16.40, \mathrm{df}=17(\mathrm{P}=0.50) ; \mathrm{I}^{2}=0 \%$

Test for overall effect: $Z=1.87(\mathrm{P}=0.06)$

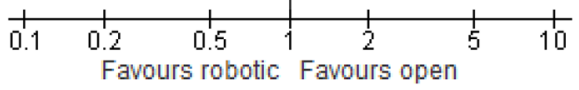

B

Robotic

Open

Odds Ratio

Study or Subgroup Events Total Events Total Weight M-H, Random, $95 \% \mathrm{Cl}$

Baker 2016

Bao 2014 (1)

Boggi 2016 (2)

Boone 2014

Buchs 2011

Butt 2016

Chalikonda 2012

Chen 2015

Girgis 2019

lelpo 2019

Kauffmann 2019

Lai 2012

Marino 2019

Shi 2020

Wang 2018

Zhou 2011

Zureikat 2016 (3)

$\begin{array}{rr}4 & 22 \\ 4 & 16 \\ 2 & 16 \\ 4 & 58 \\ 3 & 33 \\ 3 & 12 \\ 0 & 14 \\ 1 & 46 \\ 35 & 163 \\ 1 & 14 \\ 11 & 20 \\ 4 & 15 \\ 2 & 35 \\ 10 & 124 \\ 3 & 118 \\ 0 & 8 \\ 35 & 70\end{array}$

$\begin{array}{rr}14 & 49 \\ 6 & 29\end{array}$

$496.6 \%$

$0.56[0.16,1.94]$

$1.28[0.30,5.42]$

6 $11 \quad 4.0 \%$

$31 \quad 98 \quad 74 \%$

$\begin{array}{lll}5 & 27 & 5.2 \%\end{array}$

$19 \quad 55 \quad 5.7 \%$

$414 \quad 1.9 \%$

$\begin{array}{lll}4 & 14 & 1.9 \% \\ 4 & 92 & 3.1 \%\end{array}$

$43 \quad 198 \quad 11.3 \%$

$117 \quad 2.1 \%$

$10 \quad 24 \quad 6.8 \%$

$19 \quad 53 \quad 6.4 \%$

$6 \quad 35 \quad 4.7 \%$

$\begin{array}{lll}57 & 552 & 10.0 \%\end{array}$

$11 \quad 178 \quad 6.3 \%$

Total $(95 \% \mathrm{Cl})$

Total events

122

784

122

$\begin{array}{lll}1 & 6 & 1.6 \%\end{array}$

$0.12[0.02,0.79]$

$0.16[0.05,0.48]$

$0.44[0.09,2.04]$

$0.63[0.15,2.61]$

$0.08[0.00,1.66]$

$0.49[0.05,4.50]$

$0.99[0.60,1.63]$

$1.23[0.07,21.64]$

$1.71[0.52,5.67]$

$0.65[0.18,2.33]$

$0.29[0.05,1.57]$

$0.76[0.38,1.54]$

$0.40[0.11,1.45]$

$0.22[0.01,6.31]$

$2.22[1.34,3.70]$

$0.64[0.41,1.00]$

Heterogeneity: Tau $^{2}=0.40 ; \mathrm{Chi}^{2}=36.59, \mathrm{df}=16(\mathrm{P}=0.002) ; \mathrm{I}^{2}=56 \%$

Test for overall effect: $Z=1.95(P=0.05)$

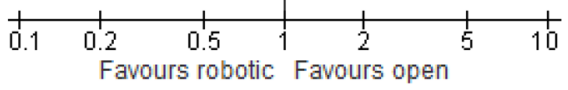

Footnotes

(1) Based on final procedure; 3 patients converted to open

(2) 16 robotic and 11 open patients had pancreatic cancer

(3) 70 robotic and 448 open procedures were for pancreatic cancer

Figure 4. Meta-analysis of primary endpoints: (A) Overall postoperative morbidity. (B) Resection margin involvement rate. 


\begin{tabular}{|l|l|l|l|l|l|}
\hline Dichotomous endpoints & RRR & ARR $(\mathbf{9 5} \% \mathbf{C I})$ & NNT (95\%CI) & $\begin{array}{l}\text { 95\% prediction } \\
\text { interval }\end{array}$ & $\begin{array}{l}\text { GRADE certainty } \\
\text { of evidence }\end{array}$ \\
\hline $\begin{array}{l}\text { Postoperative overall } \\
\text { morbidity }\end{array}$ & 0.22 & $0.118(0.086,0.149)$ & $9(7,12)$ & $0.72,1.02$ & $\begin{array}{l}\text { Moderate } \\
\oplus \oplus \oplus \bigcirc\end{array}$ \\
\hline POPF rate & 0.13 & $0.021(0.004,0.038)$ & $47(26,267)$ & $0.29,2.74$ & $\begin{array}{l}\text { Low } \\
\oplus \oplus \bigcirc \bigcirc\end{array}$ \\
\hline DGE rate & 0.03 & $0.005(-0.017,0.026)$ & $\begin{array}{l}210(>38 \text { to benefit, }>4 \text { to } \\
\text { harm })\end{array}$ & $0.51,1.87$ & $\begin{array}{l}\text { Very low } \\
\oplus \bigcirc \bigcirc\end{array}$ \\
\hline Incisional SSI rate & 0.22 & $0.022(0.004,0.040)$ & $46(25,243)$ & $0.12,1.70$ & $\begin{array}{l}\text { Low } \\
\oplus \oplus \bigcirc \bigcirc\end{array}$ \\
\hline Reoperation rate & 0.01 & $0.000(-0.013,0.014)$ & $\begin{array}{l}3,007(>76 \text { to harm, }>72 \text { to } \\
\text { benefit })\end{array}$ & $0.61,1.04$ & $\begin{array}{l}\text { Very low } \\
\oplus \bigcirc \bigcirc\end{array}$ \\
\hline Margin involvement rate & 0.22 & $0.044(0.013,0.075)$ & $23(13,79)$ & $0.15,2.68$ & $\begin{array}{l}\text { Moderate } \\
\oplus \oplus \oplus \bigcirc\end{array}$ \\
\hline Numeric endpoints & MD $(\mathbf{9 5} \% \mathbf{C I})$ & $\begin{array}{l}\text { Clinical importance of } \\
\text { the MD }\end{array}$ & Moderate & $\mathbf{9 5 \%}$ prediction interval & GRADE certainty of evidence \\
\hline Operating time & $75.17(48.05,102.28)$ & $-58.77,209.11$ & $\begin{array}{l}\text { Low } \\
\oplus \oplus \bigcirc \bigcirc\end{array}$ \\
\hline Estimated blood loss & $-191.35(-238.12,-144.59)$ & Low & Moderate & $-382.04,-0.66$ & $\begin{array}{l}\text { Moderate } \\
\oplus \oplus \oplus \bigcirc\end{array}$ \\
\hline Length of hospital stay & $-1.00(-1.88,-0.12)$ & Moderate & $-4.32,2.32$ & $\begin{array}{l}\text { Very low } \\
\oplus \bigcirc \bigcirc\end{array}$ \\
\hline $\begin{array}{l}\text { Number of lymph nodes } \\
\text { harvested }\end{array}$ & $2.88(1.12,4.65)$ & $\begin{array}{l}\text { Low } \\
\oplus \oplus \bigcirc \bigcirc\end{array}$ \\
\hline
\end{tabular}

Table 4. Clinical relevance and certainty of evidence provided by statistical difference in clinical and pathologic endpoints (dichotomous and numeric) between robotic vs. open pancreaticoduodenectomy. $R R R$ relative risk reduction; $A R R$ absolute risk reduction; NNT numbers needed to treat; $95 \% C I$ 95\% confidence interval; POPF postoperative pancreatic fistula; DGE delayed gastric emptying; SSI surgical site infection; $M D$ mean difference.

\section{Discussion}

Currently, general surgery is the fastest growing specialty for the DaVinci robotic platform in the US. Specifically, robotic PD is experiencing widespread growth since its introduction ${ }^{7}$. Similar to laparoscopic distal pancreatectomy has seen its use expanded three-fold between 1998 and 2009, robotic PD is also subject to significant growth nationwide ${ }^{56}$.

Pancreaticoduodenectomy remains a technically demanding operation with significant risks of morbidity ${ }^{57}$. Historically, minimally invasive surgery has often been compared to open approach in non-inferiority studies. With improved visualization through magnification of target anatomy and ergonomics allowing more precise excision along critical resection margins, robotic approach may allow superior dissection and skeletonization of critical borders. Margins near the uncinate process along the superior mesenteric artery requiring dissection down to the adventitia is facilitated with the robotic instruments. Although resection margins are important for overall survival and locoregional recurrence, the ability to achieve R0 resection can be as low as $60 \%$ in some open cases ${ }^{14,58}$. One of the important findings of this meta-analysis is the improvement in resection margin in robotic PD. Patients with non-involved resection margins have improved overall survival as well as decreased locoregional recurrence risk in comparison to $\mathrm{R} 1$ resection $^{14}$. The benefits of R0 resection is especially pronounced in patients with N0 disease $\mathrm{i}^{14}$.

Previously, the benefits of robotic surgery for pancreatic cancer in terms of margin status have been reported $^{12,45}$. With the high rate of locoregional failure, assessment of the circumferential margin of the Whipple specimen was re-defined in $2006^{58}$. Verbeke et al. advocated a standardized protocol for margin assessment since circumferential margin positivity can be underestimated by as much as $60 \%{ }^{59}$. Unfortunately, the method of margin quantification in the majority of these studies were not clearly defined based on the papers reviewed ${ }^{58}$. Furthermore, only two studies stated their adherence to the standardized Leeds Pathology Protocol (LEEPP) for margin assessment. Only two papers ${ }^{45,49}$ specified that the LEEPP protocol were followed. Nonetheless, Peng et al. performed a meta-analysis previously and showed improved margin status favoring robotic surgery over open surgery ${ }^{12,60}$. Within their findings, only 8 studies were included which discussed oncologic outcomes ${ }^{12}$. Kauffman et al. performed a propensity score matched analysis of robotic versus open PD and found equivalent rates of R1 resection ${ }^{45}$. The authors did comment, as speculated by many robotic surgeons, that following the peri-adventitial dissection plane close to the right side of the SMA, following early ligation of the inferior pancreaticoduodenal artery makes the retroperitoneal dissection easier. The retroperitoneal dissection plan is particularly efficient using the minimally invasive robotic approach ${ }^{45}$. In this meta-analysis, we confirmed the significant difference in resection margin involvement rates favoring robotic approach.

Similar to margin status, an increase in the number of lymph nodes harvested is frequently associated with improved staging and optimal resection margins ${ }^{17}$. In this study, we also identified that robotic PD has an increased number of lymph node harvested as compared to open. Previously, studies have shown both that total number of lymph nodes evaluated and a higher positive lymph node ratio to be superior in terms of oncological outcome. This meta-analysis is one of the first to present superior nodal sampling with the use of robotic surgery. 
A

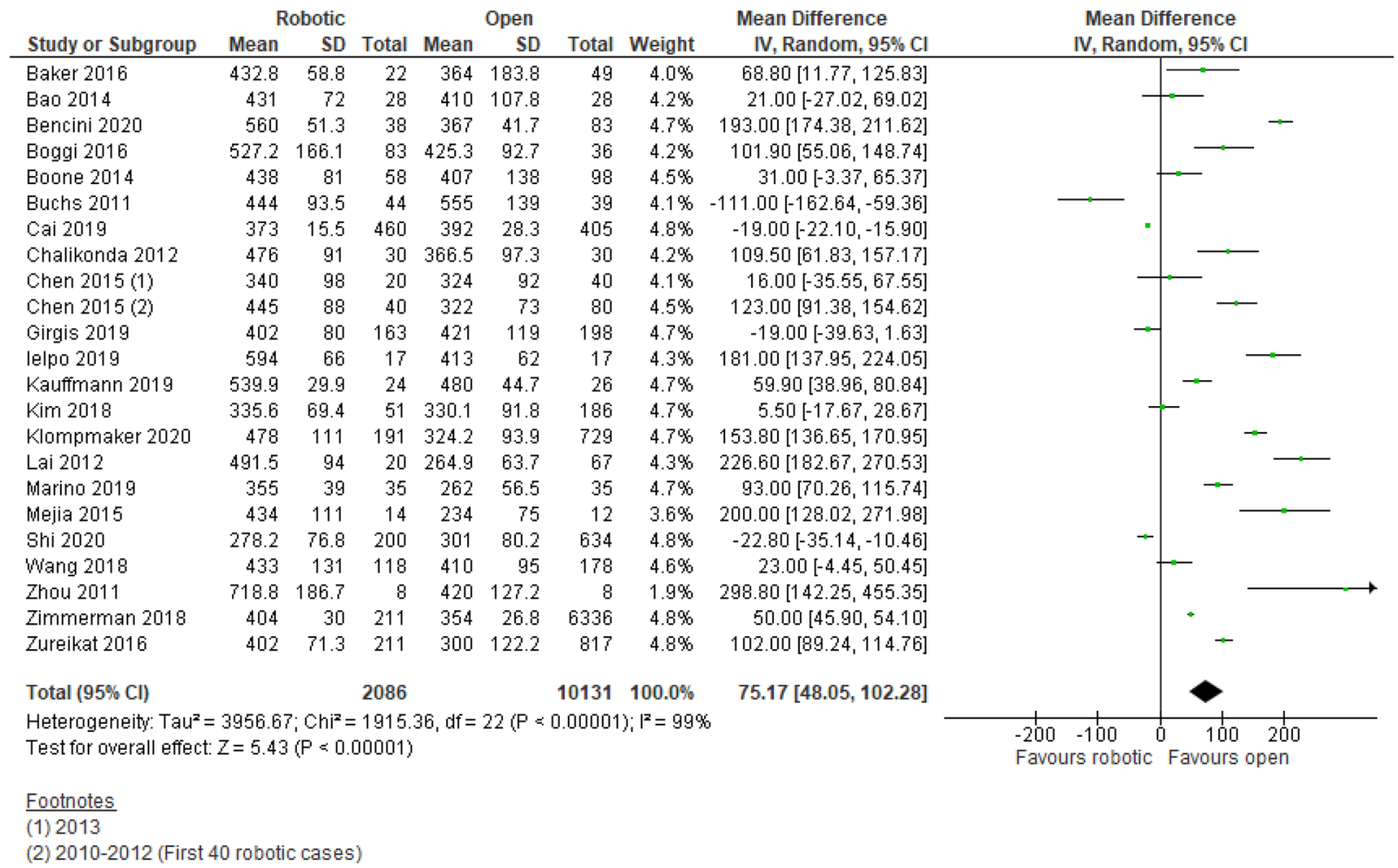

B

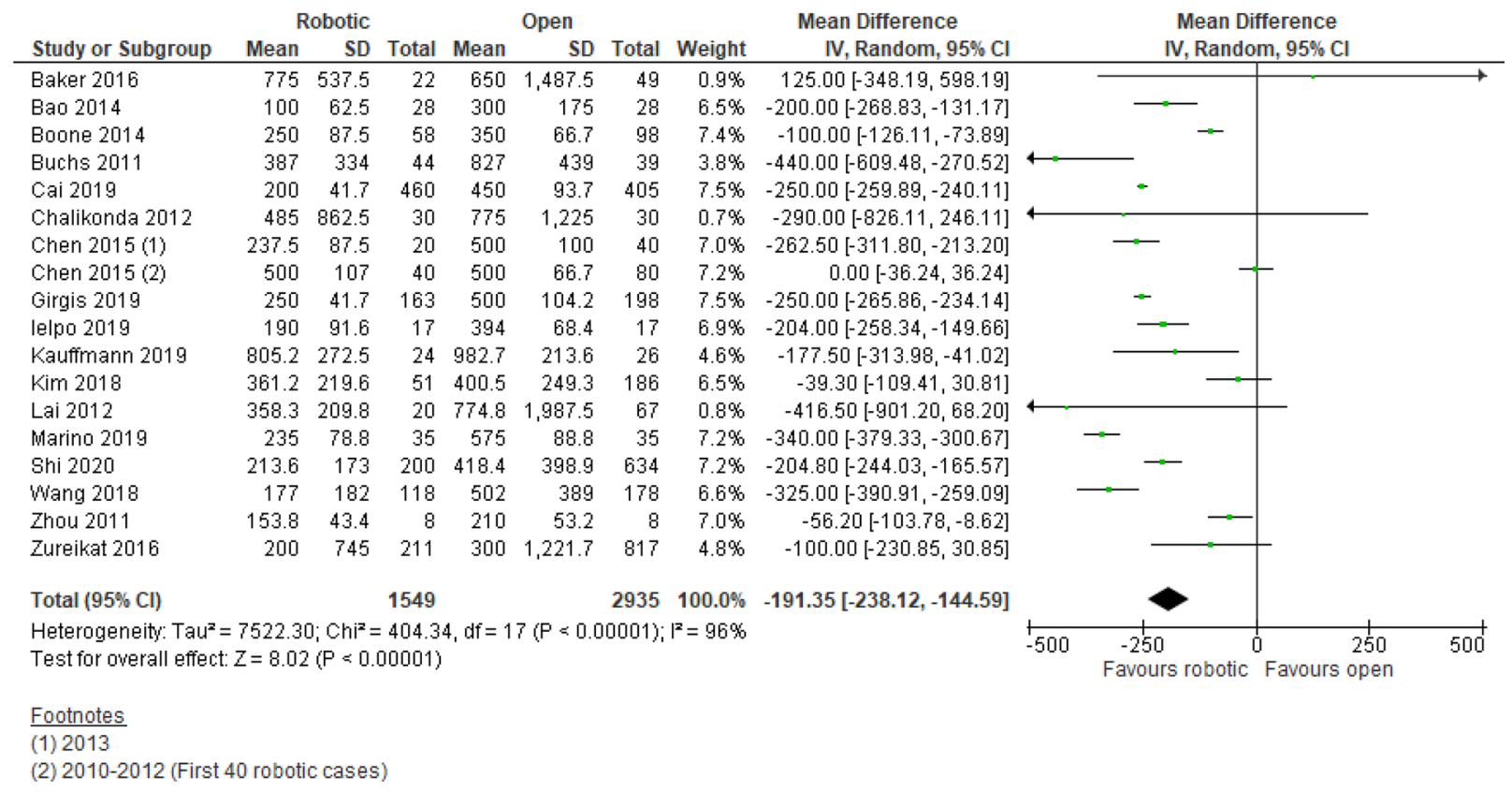

Figure 5. Meta-analysis of secondary endpoints: (A) Operating time. (B) Estimated blood loss. (C) Postoperative pancreatic fistula rate. (D) Delayed gastric emptying rate. (E) Incisional SSI rate. (F) Reoperation rate. (G) Length of hospital stay. (H) Number of lymph nodes harvested. 


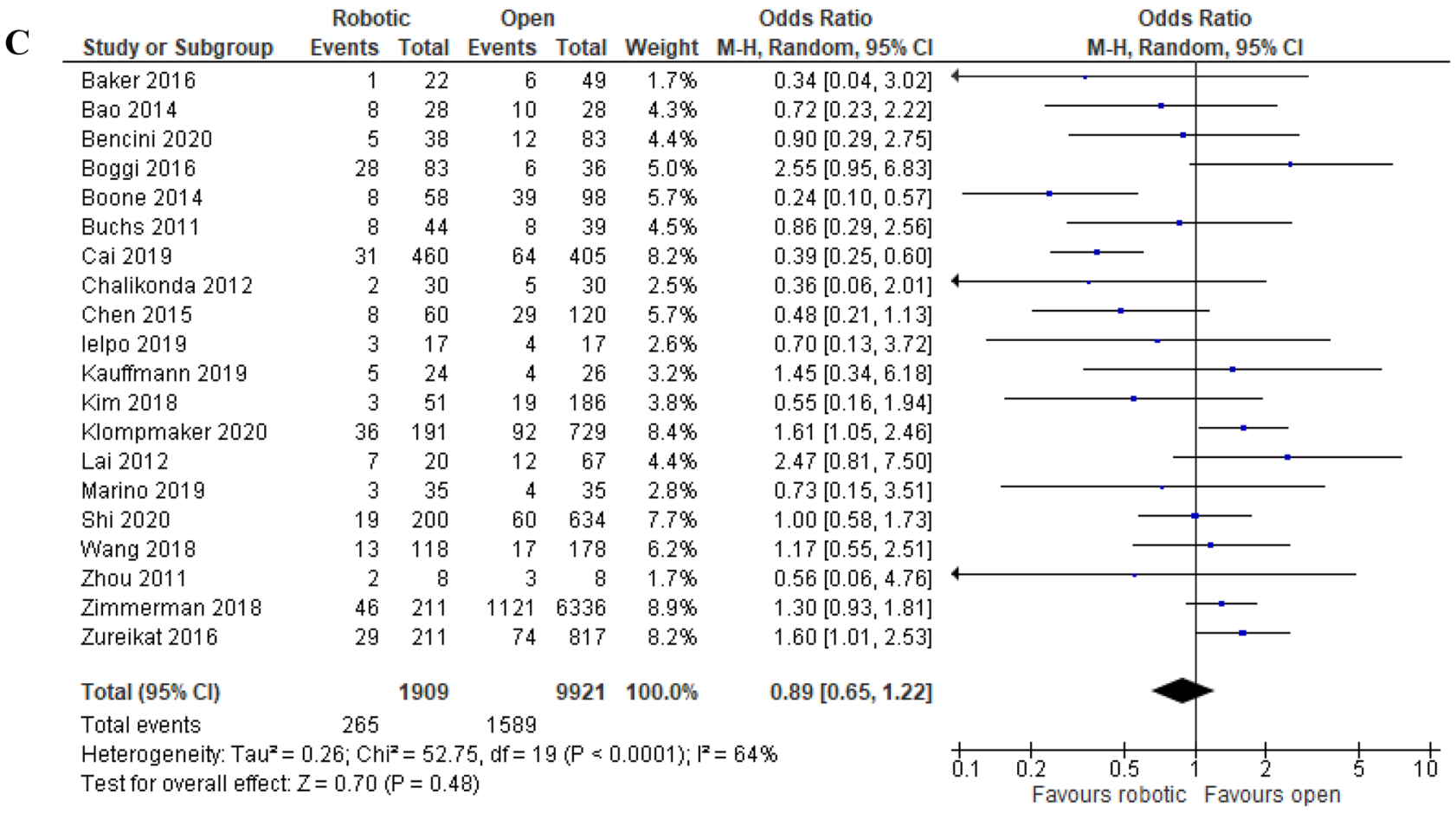

Robotic Open Odds Ratio

D Study or Subgroup Events Total Events Total Weight M-H, Random, 95\% Cl

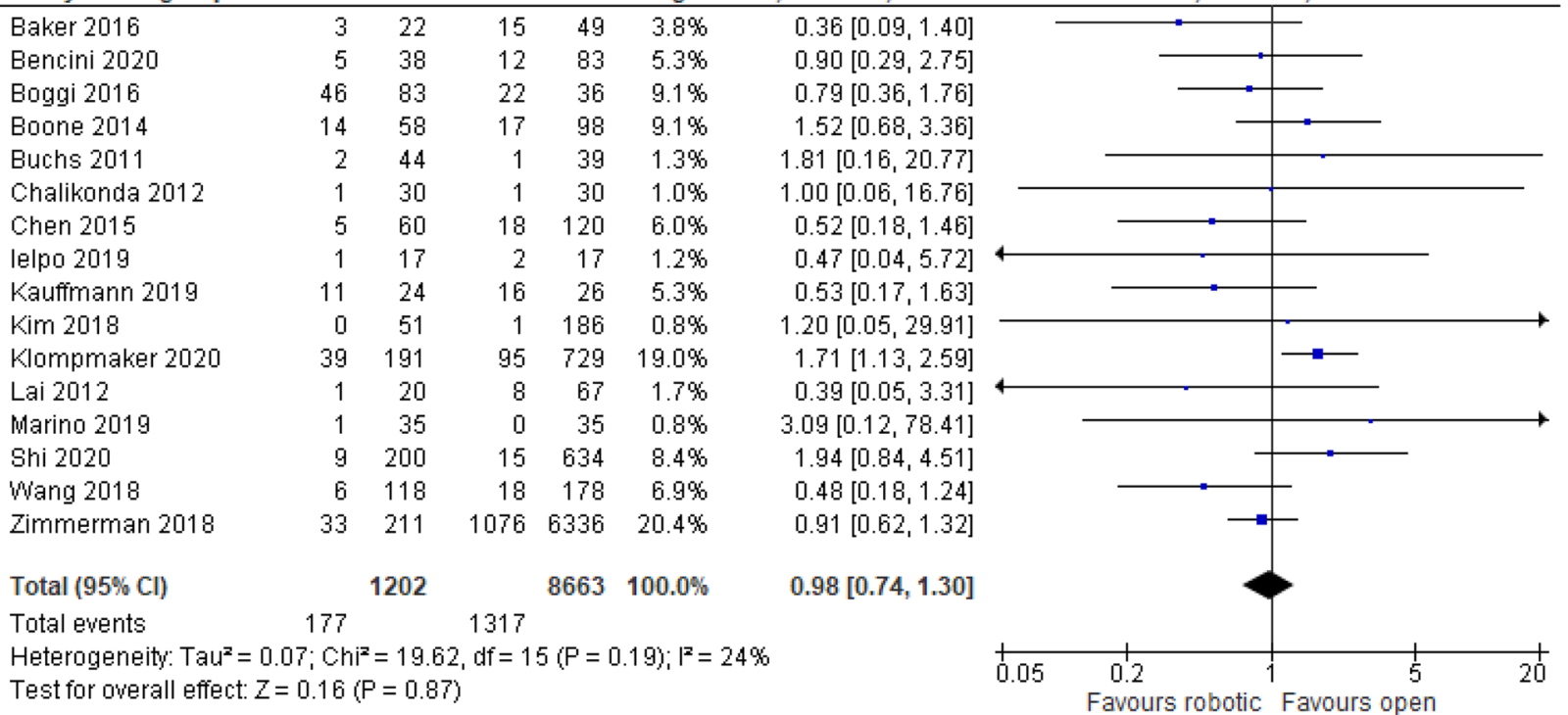

Figure 5. (continued) 
$\mathbf{E}$

\begin{tabular}{lrrrrr} 
Study or Subgroup & Events & Total & Events & Total & Weight \\
\hline Baker 2016 & 0 & 22 & 13 & 49 & $2.7 \%$ \\
Bao 2014 & 5 & 28 & 12 & 28 & $9.6 \%$ \\
Buchs 2011 & 0 & 44 & 3 & 39 & $2.5 \%$ \\
Chalikonda 2012 & 1 & 30 & 1 & 30 & $2.8 \%$ \\
Chen 2015 & 1 & 60 & 15 & 120 & $4.7 \%$ \\
Girgis 2019 & 24 & 163 & 71 & 198 & $18.0 \%$ \\
lelpo 2019 & 0 & 17 & 4 & 17 & $2.5 \%$ \\
Kim 2018 & 1 & 51 & 4 & 186 & $4.2 \%$ \\
Lai 2012 & 1 & 20 & 4 & 67 & $4.1 \%$ \\
Marino 2019 & 0 & 35 & 2 & 35 & $2.4 \%$ \\
Wang 2018 & 3 & 118 & 14 & 178 & $9.2 \%$ \\
Zimmerman 2018 & 20 & 211 & 568 & 6336 & $18.7 \%$ \\
Zureikat 2016 & 24 & 211 & 109 & 817 & $18.6 \%$ \\
Total (95\% Cl) & & & & & \\
Total events & & 1010 & & 8100 & $100.0 \%$ \\
Ho & 80 & & 920 & &
\end{tabular}

Heterogeneity: Tau $^{2}=0.30 ; \mathrm{Chi}^{2}=25.21, \mathrm{df}=12(\mathrm{P}=0.01) ; \mathrm{I}^{2}=52 \%$

Test for owerall effect: $Z=3.14(\mathrm{P}=0.002)$
Odds Ratio

, Random, $95 \% \mathrm{Cl}$ $0.06[0.00,1.06]$ $0.29[0.09,0.98]$ $0.12[0.01,2.34]$ $1.00[0.06,16.76]$ $0.12[0.02,0.92]$ $0.31[0.18,0.52]$ $0.09[0.00,1.73]$ $0.91[0.10,8.32]$ $0.83[0.09,7.87]$ $0.19[0.01,4.08]$ $0.31[0.09,1.09]$ $1.06[0.67,1.70]$ $0.83[0.52,1.33]$

$0.45[0.27,0.74]$

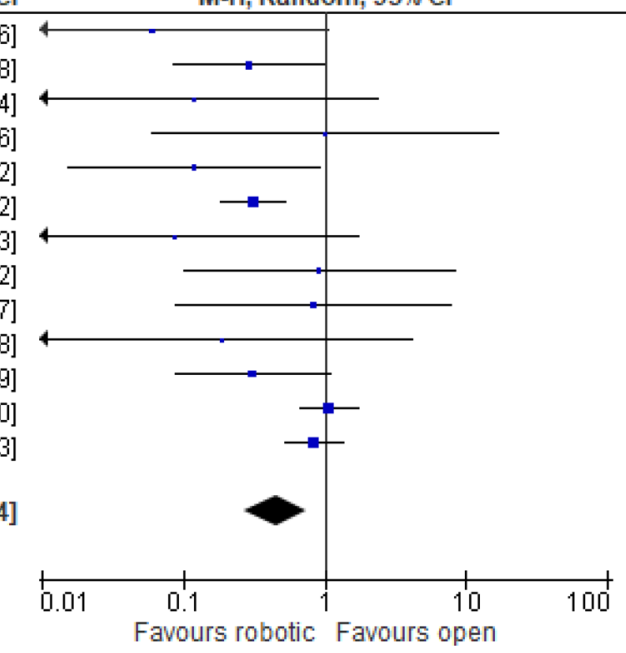

F

Robotic Open Odds Ratio Study or Subgroup Events Total Events Total Weight M-H, Random, 95\% Cl $\begin{array}{lllllll}\text { Baker 2016 } & 1 & 22 & 6 & 49 & 1.3 \% & 0.34[0.04,3.02]\end{array}$ Bao 2014

Bencini 2020

Boggi 2016

Boone 2014

Buchs 2011

Cai 2019

Chalikonda 2012

Chen 2015

leipo 2019

Kauffmann 2019

Klompmaker 2020

Lai 2012

Marino 2019

Shi 2020

Zimmerman 2018

$\begin{array}{ll}3 & 28 \\ 8 & 38\end{array}$

$4 \quad 28 \quad 2.4 \%$

$0.72[0.15,3.56]$

$14 \quad 83 \quad 6.5 \%$

$1.31[0.50,3.46]$

$11 \quad 83$

$4 \quad 36 \quad 4.1 \%$

$\begin{array}{lllll}1 & 58 \quad 8 & 98 & 1.4 \%\end{array}$

$1.22[0.36,4.13]$

$0.20[0.02,1.62]$

$\begin{array}{lllll}2 & 44 & 5 & 39 & 2.1 \%\end{array}$

$\begin{array}{rr}2 & 44 \\ 19 & 460\end{array}$

$\begin{array}{rrr}32 & 405 & 18.0 \%\end{array}$

$0.32[0.06,1.77]$

$0.50[0.28,0.90]$

$0.37[0.08,1.58]$

$1.00[0.18,5.62]$

$\begin{array}{lllll}2 & 60 & 4 & 120 & 2.1 \%\end{array}$

Total $(95 \% \mathrm{Cl})$

$3 \quad 17$

$\begin{array}{rrr}4 & 120 & 2.1 \% \\ 2 & 17 & 1.6 \%\end{array}$

$1.61[0.23,11.09]$

$3.38[0.13,87.11]$

$0.88[0.53,1.45]$

$21 \quad 191$

$\begin{array}{lll}0 & 26 & 0.6 \%\end{array}$

$2.37[0.37,15.29]$

$0.65[0.10,4.13]$

$0.52[0.23,1.19]$

$1.20[0.69,2.09]$

Total events 100

1521

$41 \quad 634 \quad 9.2 \%$

$0.80[0.62,1.02]$

Heterogeneity: $\operatorname{Tau}^{2}=0.00 ; \mathrm{Chi}^{2}=14.38, \mathrm{df}=15(\mathrm{P}=0.50) ; \mathrm{I}^{2}=0 \%$

Test for owerall effect: $Z=1.79(\mathrm{P}=0.07)$

$8732 \quad 100.0 \%$

(a)

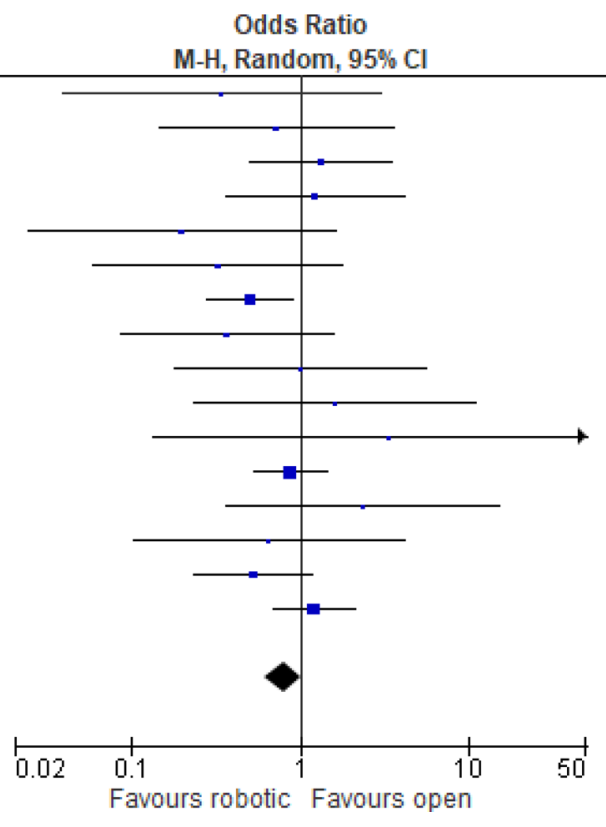

Figure 5. (continued) 
G

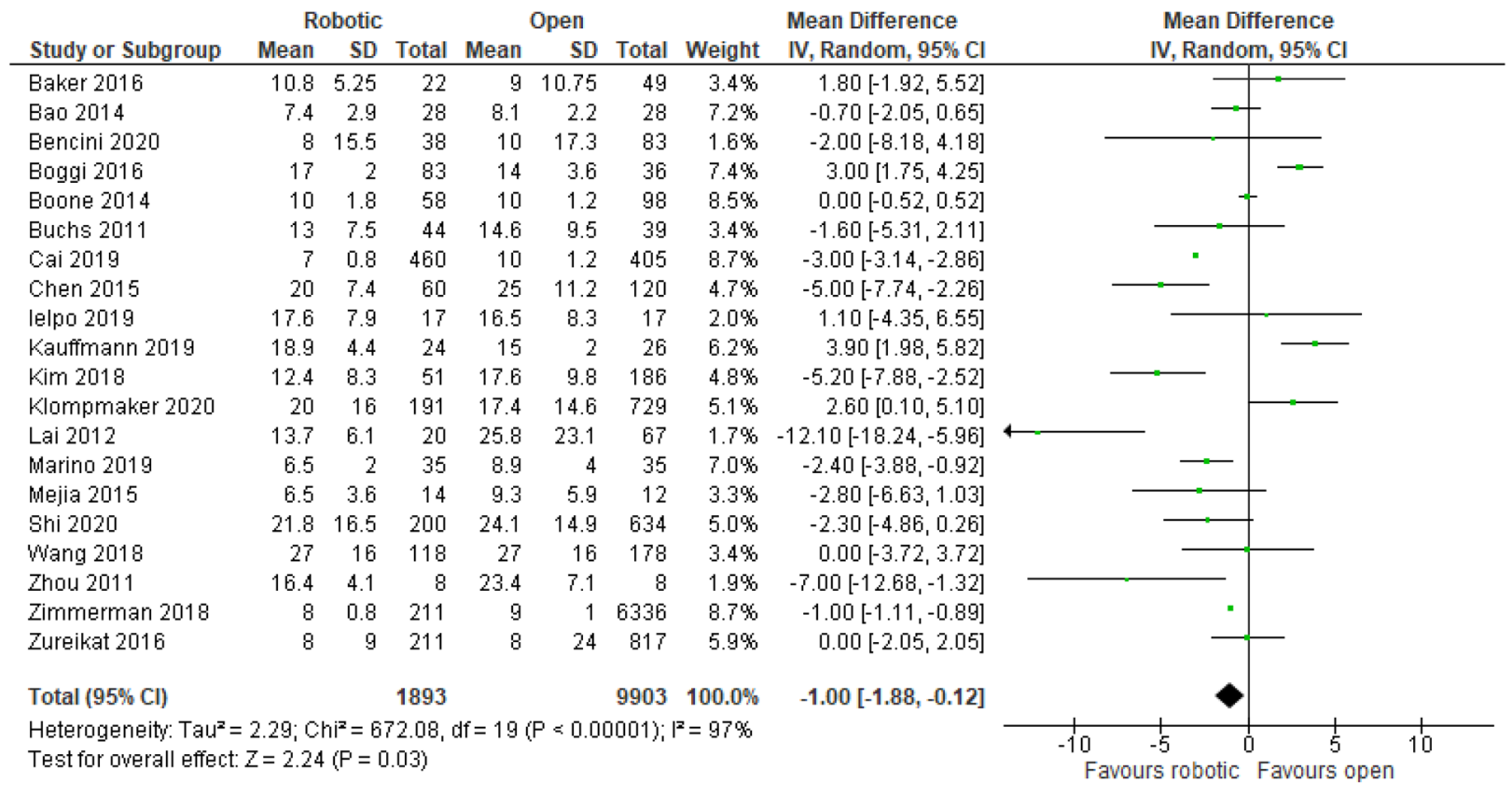

H

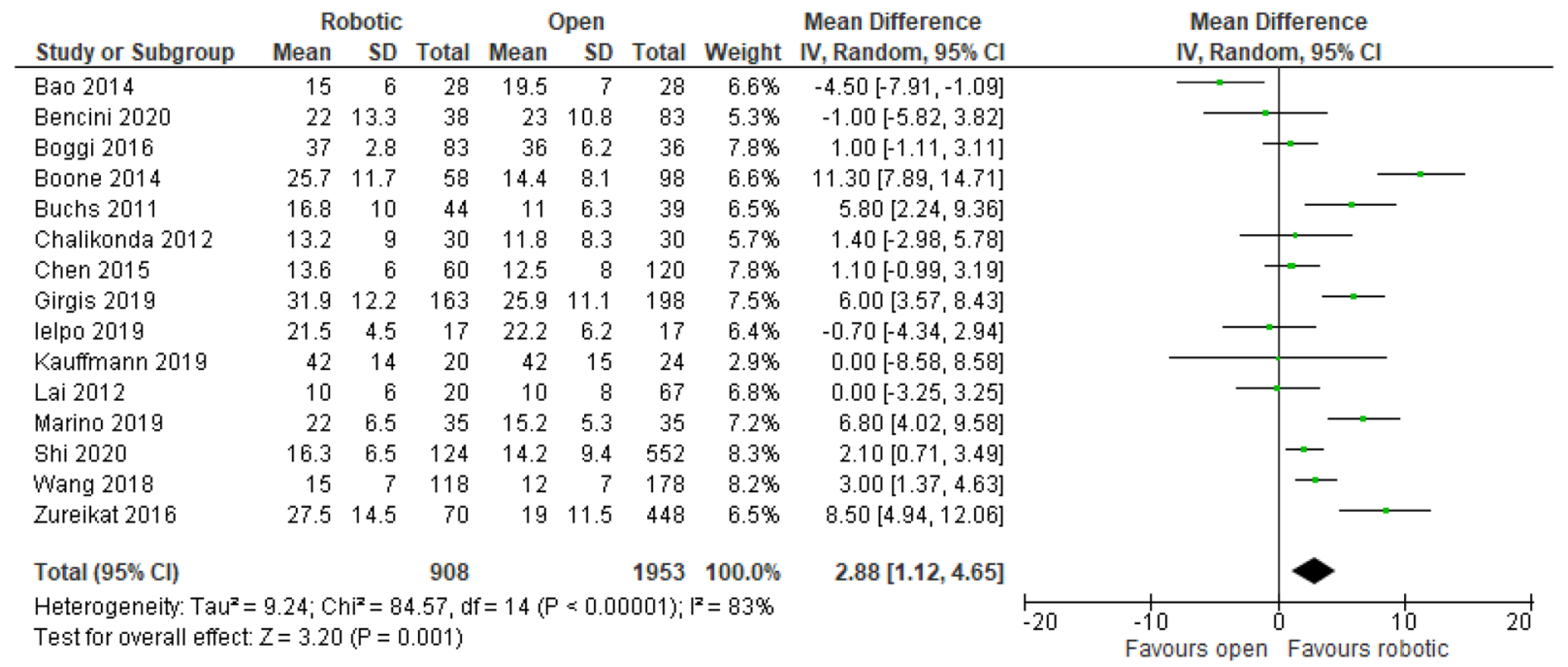

Figure 5. (continued) 


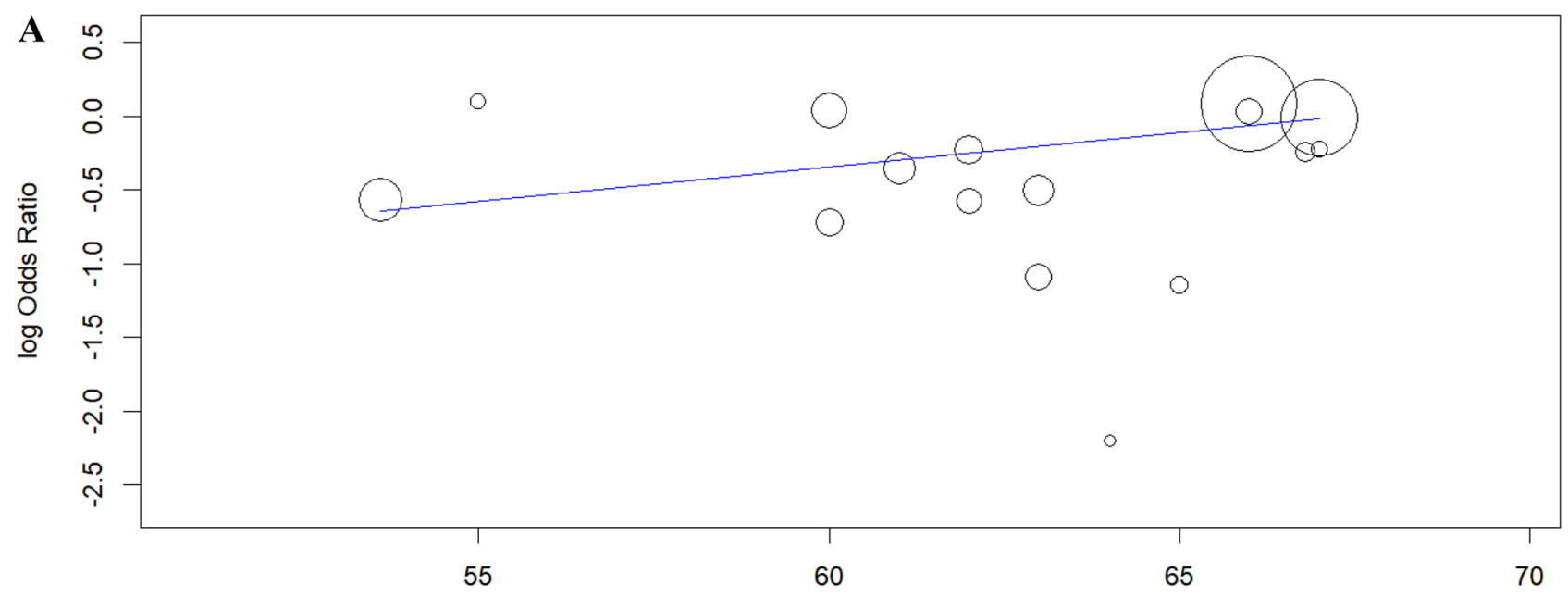

Age (robotic PD)

B

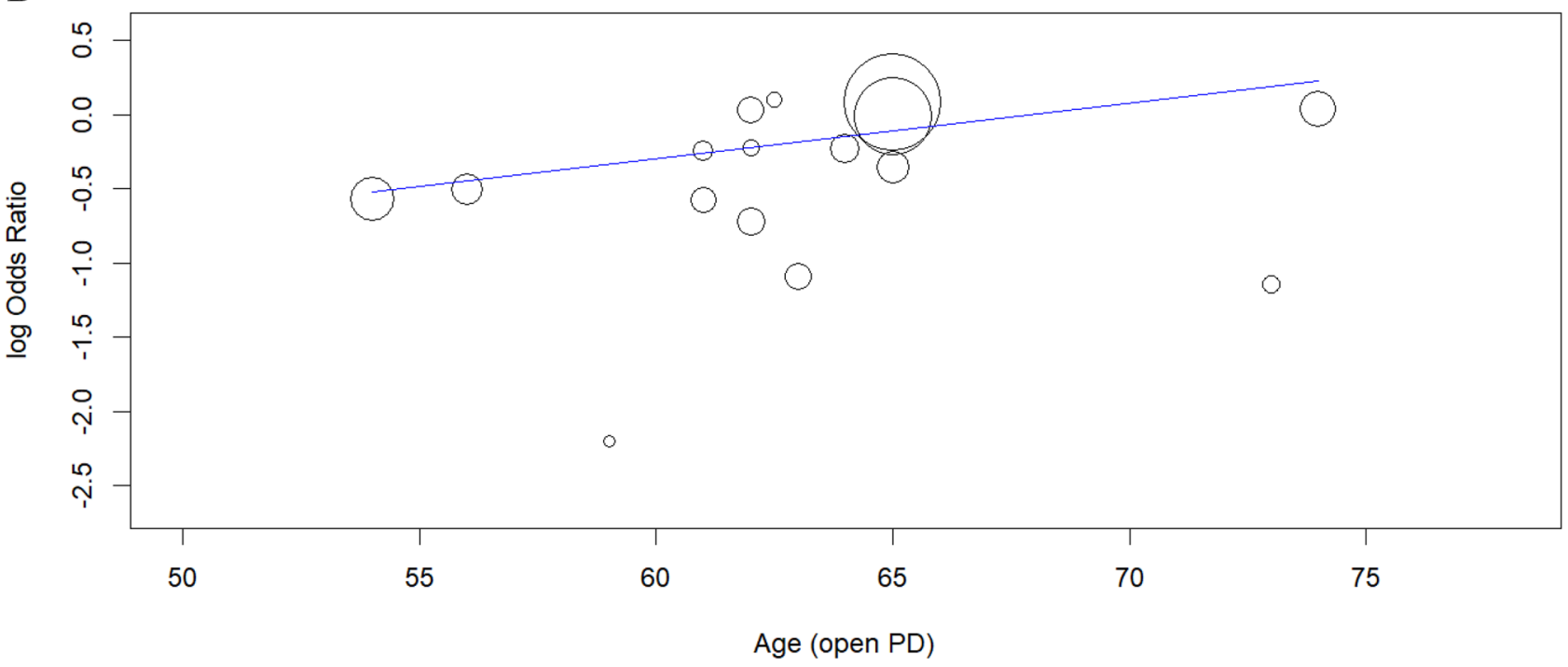

Figure 6. Ad-hoc meta-regression analysis: (A) Regression plot of overall postoperative morbidity and average age in robotic $\mathrm{PD}$ (Omnibus $\mathrm{p}=0.040$ ). (B) Regression plot of overall postoperative morbidity and average age in open PD (Omnibus $\mathrm{p}=0.075)$.

As expected, clinical outcomes favoring robotic surgery included significantly lower estimated blood loss, decreased incisional SSI rate, and lower length of hospital stay at the cost of longer operating time. These findings confirmed the results of previous meta-analyses. No significant difference in POPF, DGE, and reoperation rates was found.

One of the strengths of this meta-analysis is the number of studies and thereby number of patients included. Other strengths were prospective development and registration of the protocol, and rigorous literature search. This meta-analysis has several limitations. Given the observational nature, all included studies were subject to high risk of selection, performance, and detection biases. Moreover, all studies reported only short-term outcomes. The differences in surgical approaches and perioperative management across the globe may have contributed further to the heterogeneity and variance across the included studies. The lack of other histopathological details including and not limited to lymphovascular and perineural invasion adds additional heterogeneity. Another limitation was a lack of standardization in the definitions of interventions, a fact that may have contributed to the risk of performance bias. 

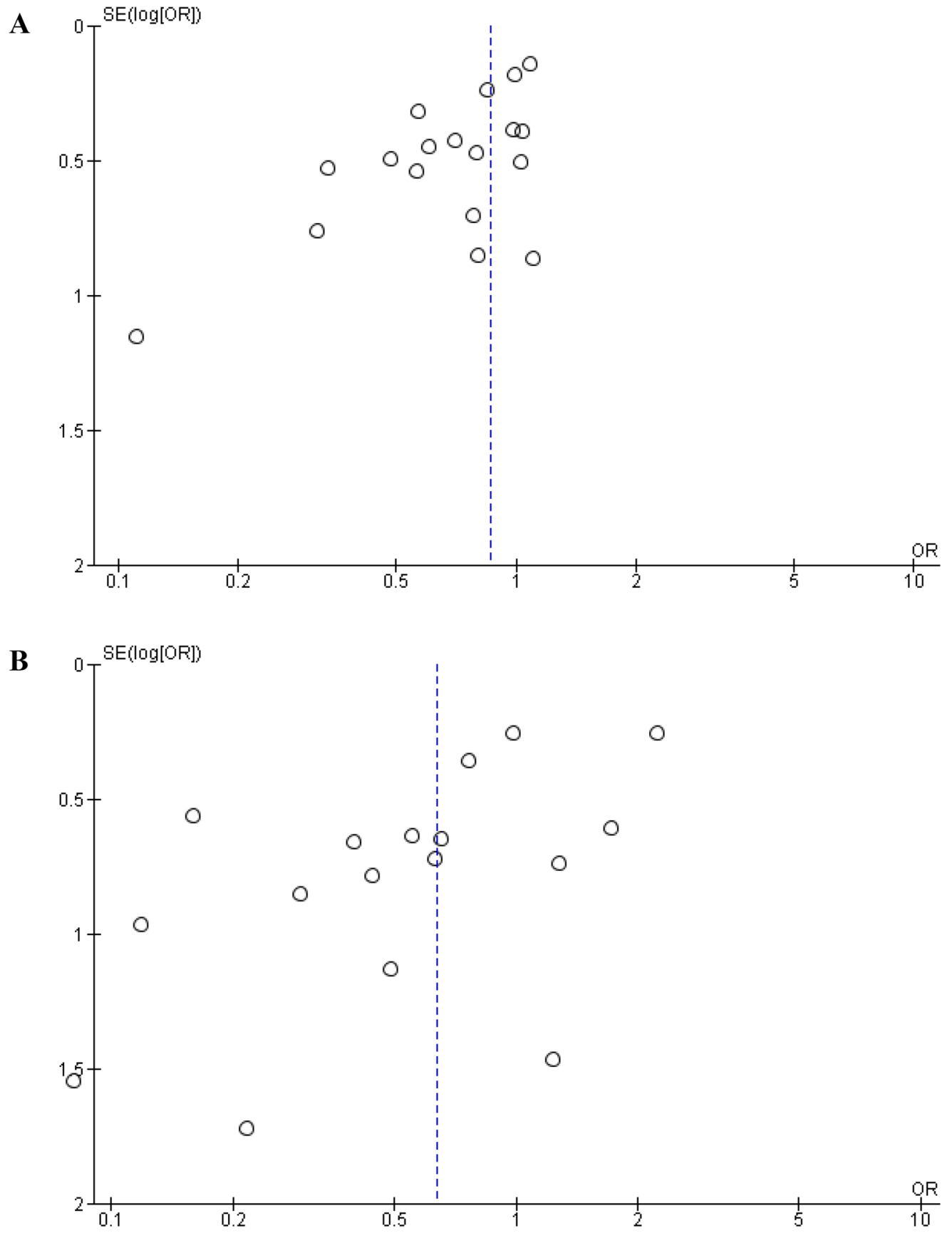

Figure 7. Funnel plot of reporting bias: (A) Overall postoperative morbidity. (B) Resection margin involvement rate.

\section{Conclusion}

This meta-analysis found that robotic PD was associated with improved resection margins and number of lymph node harvested as compared to open PD. Moreover, robotic PD allowed surgery with less blood loss and was associated with decreased wound infection rates and shorter length of hospital stay, at the expense of increased operating time and surgical cost. The current application of robotic PD needs further experimental and observational prospective studies given the possible benefits over open PD. 
A

\begin{tabular}{llll} 
Studies & \multicolumn{2}{c}{ Estimate $(95 \%$ C.I.) } \\
Overall & 0.861 & $(0.736,1.007)$ \\
- Baker & 0.880 & $(0.751,1.032)$ \\
- Bencini & 0.850 & $(0.722,1.000)$ \\
- Boggi & 0.854 & $(0.725,1.007)$ \\
- Boone & 0.849 & $(0.720,1.001)$ \\
- Buchs & 0.871 & $(0.743,1.022)$ \\
- Chalikonda & 0.869 & $(0.742,1.019)$ \\
- Chen & 0.886 & $(0.753,1.042)$ \\
- lelpo & 0.853 & $(0.725,1.005)$ \\
- Hammill & 0.852 & $(0.724,1.002)$ \\
- Kauffmann & 0.871 & $(0.744,1.020)$ \\
- Kim & 0.864 & $(0.734,1.016)$ \\
- Lai & 0.850 & $(0.722,1.001)$ \\
- Marino & 0.874 & $(0.746,1.025)$ \\
- Mejia & 0.853 & $(0.724,1.004)$ \\
- Wang & 0.852 & $(0.716,1.013)$ \\
- Zhou & 0.870 & $(0.743,1.018)$ \\
- Zimmerman & 0.777 & $(0.643,0.938)$ \\
- Zureikat & 0.833 & $(0.699,0.992)$
\end{tabular}

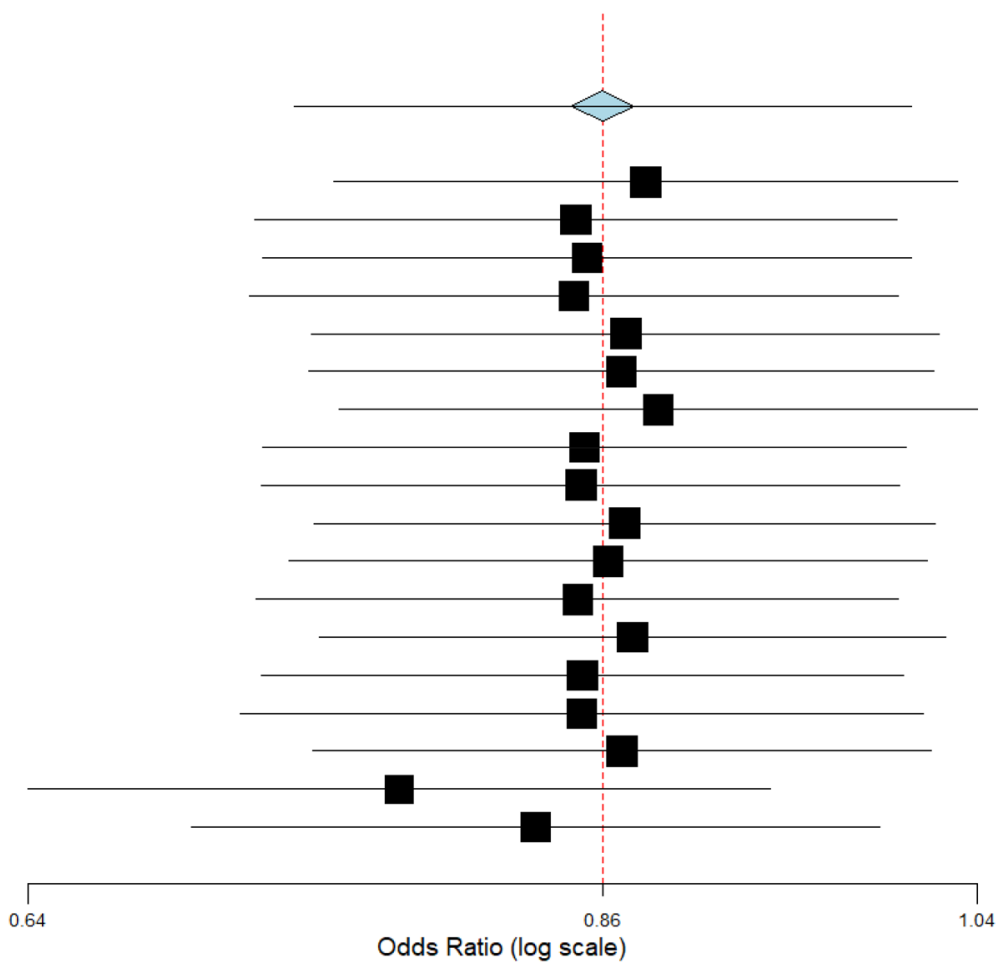

\section{B}

\begin{tabular}{llll} 
Studies & \multicolumn{3}{c}{ Estimate $(95 \%$ C.I. $)$} \\
Overall & 0.853 & $(0.551,1.320)$ \\
& & & \\
- Baker & 0.875 & $(0.553,1.385)$ \\
- Bao & 0.822 & $(0.517,1.307)$ \\
- Boggi & 0.950 & $(0.630,1.431)$ \\
- Boone & 0.816 & $(0.513,1.300)$ \\
- Buchs & 0.884 & $(0.563,1.387)$ \\
- Butt & 0.794 & $(0.513,1.231)$ \\
- Chalikonda & 0.900 & $(0.588,1.380)$ \\
- Chen & 0.864 & $(0.550,1.355)$ \\
- Girgis & 0.798 & $(0.494,1.291)$ \\
- lelpo & 0.840 & $(0.535,1.318)$ \\
- Kauffmann & 0.800 & $(0.502,1.274)$ \\
- Lai & 0.862 & $(0.543,1.369)$ \\
- Marino & 0.905 & $(0.583,1.404)$ \\
- Shi & 0.848 & $(0.522,1.377)$ \\
- Wang & 0.904 & $(0.578,1.413)$ \\
- Zhou & 0.870 & $(0.559,1.353)$ \\
- Zureikat & 0.769 & $(0.519,1.138)$
\end{tabular}

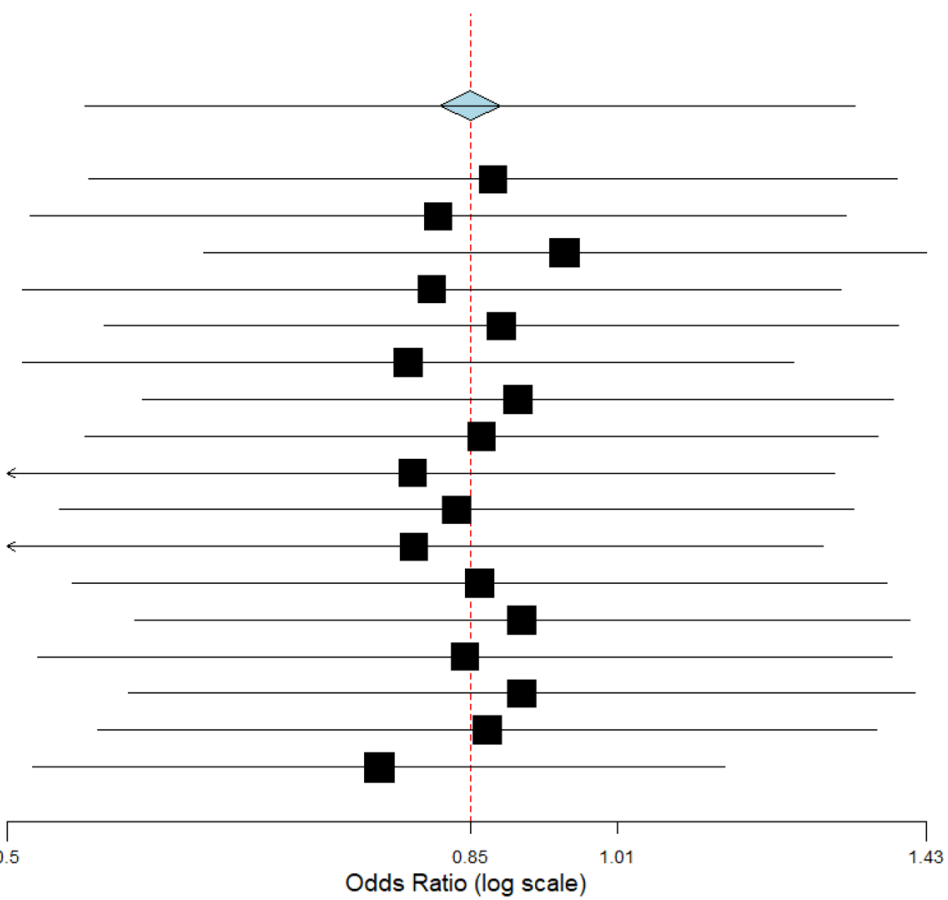

Figure 8. Sensitivity analysis: (A) Leave-one-out meta-analysis of overall postoperative morbidity. (B) Leaveone-out meta-analysis of margin involvement rate.

\section{Data availability}

The datasets generated during and/or analyzed during the current study are available from the corresponding author on reasonable request.

Received: 19 June 2020; Accepted: 1 February 2021

Published online: 12 February 2021 


\section{References}

1. Cirocchi, R. et al. A systematic review on robotic pancreaticoduodenectomy. Surg. Oncol. 22, 238-246. https://doi.org/10.1016/j. suronc.2013.08.003 (2013).

2. Neoptolemos, J. P. et al. Therapeutic developments in pancreatic cancer: current and future perspectives. Nat. Rev. Gastroenterol. Hepatol. 15, 333-348. https://doi.org/10.1038/s41575-018-0005-x (2018).

3. Stafford, A. T. \& Walsh, R. M. Robotic surgery of the pancreas: The current state of the art. J. Surg. Oncol. 112, 289-294. https:// doi.org/10.1002/jso.23952 (2015).

4. Mabrut, J. Y. et al. Laparoscopic pancreatic resection: Results of a multicenter European study of 127 patients. Surgery 137, 597-605. https://doi.org/10.1016/j.surg.2005.02.002 (2005).

5. Boggi, U. et al. Laparoscopic pancreaticoduodenectomy: A systematic literature review. Surg. Endosc. 29, 9-23. https://doi. org/10.1007/s00464-014-3670-z (2015).

6. Gagner, M. \& Pomp, A. Laparoscopic pylorus-preserving pancreatoduodenectomy. Surg. Endosc. 8, 408-410. https://doi. org/10.1007/bf00642443 (1994).

7. Giulianotti, P. C. et al. Robotics in general surgery: Personal experience in a large community hospital. Arch. Surg. 138, 777-784. https://doi.org/10.1001/archsurg.138.7.777 (2003).

8. Zureikat, A. H. et al. 250 robotic pancreatic resections: safety and feasibility. Ann. Surg. 258, 554-559. https://doi.org/10.1097/ SLA.0b013e3182a4e87c (2013) (discussion 559-562).

9. Adam, M. A. et al. Minimally invasive versus open pancreaticoduodenectomy for cancer: Practice patterns and short-term outcomes among 7061 patients. Ann. Surg. 262, 372-377. https://doi.org/10.1097/SLA.0000000000001055 (2015).

10. Torphy, R. J. et al. Comparing short-term and oncologic outcomes of minimally invasive versus open pancreaticoduodenectomy across low and high volume centers. Ann. Surg. 270, 1147-1155. https://doi.org/10.1097/SLA.0000000000002810 (2019).

11. Zhao, W. et al. Safety and efficacy for robot-assisted versus open pancreaticoduodenectomy and distal pancreatectomy: A systematic review and meta-analysis. Surg. Oncol. 27, 468-478. https://doi.org/10.1016/j.suronc.2018.06.001 (2018).

12. Peng, L., Lin, S., Li, Y. \& Xiao, W. Systematic review and meta-analysis of robotic versus open pancreaticoduodenectomy. Surg. Endosc. 31, 3085-3097. https://doi.org/10.1007/s00464-016-5371-2 (2017).

13. Nickel, F. et al. Laparoscopic versus open pancreaticoduodenectomy: A systematic review and meta-analysis of randomized controlled trials. Ann. Surg. 271, 54-66. https://doi.org/10.1097/SLA.0000000000003309 (2020).

14. Tummers, W. S. et al. Impact of resection margin status on recurrence and survival in pancreatic cancer surgery. Br. J. Surg. 106, 1055-1065. https://doi.org/10.1002/bjs.11115 (2019).

15. Bilimoria, K. Y. et al. Effect of hospital volume on margin status after pancreaticoduodenectomy for cancer. J Am Coll Surg 207, 510-519. https://doi.org/10.1016/j.jamcollsurg.2008.04.033 (2008).

16. Winter, J. M. et al. 1423 pancreaticoduodenectomies for pancreatic cancer: A single-institution experience. J. Gastrointest. Surg. 10, 1199-1210. https://doi.org/10.1016/j.gassur.2006.08.018 (2006) (discussion 1210-1191).

17. You, M. S. et al. Lymph node ratio as valuable predictor in pancreatic cancer treated with R0 resection and adjuvant treatment. BMC Cancer 19, 952. https://doi.org/10.1186/s12885-019-6193-0 (2019).

18. Higgins, J. P. Cochrane Handbook for Systematic Reviews of Interventions (Wiley, New York, 2011).

19. Moher, D., Liberati, A., Tetzlaff, J., Altman, D. G. \& Group, P. Preferred reporting items for systematic reviews and meta-analyses: The PRISMA statement. Int J Surg 8, 336-341. https://doi.org/10.1016/j.ijsu.2010.02.007 (2010).

20. Stroup, D. F. et al. Meta-analysis of observational studies in epidemiology: A proposal for reporting. Meta-analysis Of Observational Studies in Epidemiology (MOOSE) group. JAMA 283, 2008-2012. https://doi.org/10.1001/jama.283.15.2008 (2000).

21. Dindo, D., Demartines, N. \& Clavien, P. A. Classification of surgical complications: A new proposal with evaluation in a cohort of 6336 patients and results of a survey. Ann. Surg. 240, 205-213 (2004).

22. Horan, T. C., Gaynes, R. P., Martone, W. J., Jarvis, W. R. \& Emori, T. G. CDC definitions of nosocomial surgical site infections, 1992: A modification of CDC definitions of surgical wound infections. Am. J. Infect. Control 20, 271-274. https://doi.org/10.1016/ s0196-6553(05)80201-9 (1992)

23. Sterne, J. A. et al. ROBINS-I: A tool for assessing risk of bias in non-randomised studies of interventions. BMJ 355, i4919. https:// doi.org/10.1136/bmj.i4919(2016).

24. Hozo, S. P., Djulbegovic, B. \& Hozo, I. Estimating the mean and variance from the median, range, and the size of a sample. BMC Med. Res. Methodol. 5, 13. https://doi.org/10.1186/1471-2288-5-13 (2005).

25. Higgins, J. P., Thompson, S. G., Deeks, J. J. \& Altman, D. G. Measuring inconsistency in meta-analyses. BMJ 327, 557-560. https ://doi.org/10.1136/bmj.327.7414.557 (2003).

26. IntHout, J., Ioannidis, J. P., Rovers, M. M. \& Goeman, J. J. Plea for routinely presenting prediction intervals in meta-analysis. BMJ Open 6, e010247. https://doi.org/10.1136/bmjopen-2015-010247 (2016)

27. Napoli, N. et al. Robotic versus open pancreatoduodenectomy: A propensity score-matched analysis based on factors predictive of postoperative pancreatic fistula. Surg. Endosc. 32, 1234-1247. https://doi.org/10.1007/s00464-017-5798-0 (2018).

28. Boggi, U. et al. Robotic-Assisted Pancreatic Resections. World J. Surg. 40, 2497-2506. https://doi.org/10.1007/s00268-016-3565-3 (2016).

29. Walsh, M. et al. Laparoscopic robotic assisted Whipple: Early results of a novel technique and comparison with the standard open procedure. Surg. Endosc. 25, S221 (2011).

30. Chalikonda, S., Aguilar-Saavedra, J. R. \& Walsh, R. M. Laparoscopic robotic-assisted pancreaticoduodenectomy: A case-matched comparison with open resection. Surg. Endosc. 26, 2397-2402. https://doi.org/10.1007/s00464-012-2207-6 (2012).

31. McMillan, M. T. et al. A propensity score-matched analysis of robotic vs open pancreatoduodenectomy on incidence of pancreatic fistula. JAMA Surg. 152, 327-335. https://doi.org/10.1001/jamasurg.2016.4755 (2017).

32. Varley, P. R. et al. Does robotic pancreaticoduodenectomy improve outcomes in patients with high risk morphometric features compared to the open approach. HPB (Oxford) 21, 695-701. https://doi.org/10.1016/j.hpb.2018.10.016 (2019).

33. Wilson, G. C. et al. Robotic approach mitigates the effect of major complications on survival after pancreaticoduodenectomy for periampullary cancer. HPB 21, S125-S126 (2019).

34. Boone, B. A. et al. Comparison of robot versus open pancreaticoduodenectomy. HPB 16(Suppl1), 91 (2014).

35. Cai, J. et al. Robotic pancreaticoduodenectomy is associated with decreased clinically relevant pancreatic fistulas: A propensitymatched analysis. J. Gastrointest. Surg. https://doi.org/10.1007/s11605-019-04274-1 (2019).

36. Baker, E. H. et al. Robotic pancreaticoduodenectomy: Comparison of complications and cost to the open approach. Int. J. Med. Robot. 12, 554-560. https://doi.org/10.1002/rcs.1688 (2016).

37. Bao, P. Q., Mazirka, P. O. \& Watkins, K. T. Retrospective comparison of robot-assisted minimally invasive versus open pancreaticoduodenectomy for periampullary neoplasms. J. Gastrointest. Surg. 18, 682-689. https://doi.org/10.1007/s11605-013-2410-3 (2014).

38. Bencini, L. et al. Single-centre comparison of robotic and open pancreatoduodenectomy: A propensity score-matched study. Surg. Endosc. https://doi.org/10.1007/s00464-019-07335-3 (2020).

39. Buchs, N. C. et al. Robotic versus open pancreaticoduodenectomy: A comparative study at a single institution. World J. Surg. 35, 2739-2746. https://doi.org/10.1007/s00268-011-1276-3 (2011).

40. Butt, M. U. et al. Is robotic pancreaticoduodenectomy for you? Defying the steep learning curve!. HPB 18(S1), e1-e384 (2016). 
41. Chen, S. et al. Robot-assisted laparoscopic versus open pancreaticoduodenectomy: A prospective, matched, mid-term follow-up study. Surg. Endosc. 29, 3698-3711. https://doi.org/10.1007/s00464-015-4140-y (2015).

42. Girgis, M. D. et al. Oncologic outcomes after robotic pancreatic resections are not inferior to open surgery. Ann. Surg. https://doi. org/10.1097/SLA.0000000000003615 (2019).

43. Hammill, C. et al. Robotic assistance may provide the technical capability to perform a safe, minimally invasive pancreaticoduodenectomy. HPB 12, 198 (2010).

44. Ielpo, B. et al. Robotic versus standard open pancreatectomy: A propensity score-matched analysis comparison. Updates Surg. 71, 137-144. https://doi.org/10.1007/s13304-018-0529-1 (2019).

45. Kauffmann, E. F. et al. A propensity score-matched analysis of robotic versus open pancreatoduodenectomy for pancreatic cancer based on margin status. Surg. Endosc. 33, 234-242. https://doi.org/10.1007/s00464-018-6301-2 (2019).

46. Kim, H. S. et al. Comparison of surgical outcomes between open and robot-assisted minimally invasive pancreaticoduodenectomy. J. Hepatobiliary Pancreat. Sci. 25, 142-149. https://doi.org/10.1002/jhbp.522 (2018).

47. Klompmaker, S. et al. Outcomes after minimally-invasive versus open pancreatoduodenectomy: A Pan-European propensity score matched STUDY. Ann. Surg. 271, 356-363. https://doi.org/10.1097/SLA.0000000000002850 (2020).

48. Lai, E. C., Yang, G. P. \& Tang, C. N. Robot-assisted laparoscopic pancreaticoduodenectomy versus open pancreaticoduodenectomy: A comparative study. Int. J. Surg. 10, 475-479. https://doi.org/10.1016/j.ijsu.2012.06.003 (2012).

49. Marino, M. V. et al. Robotic-assisted versus open pancreaticoduodenectomy: The results of a case-matched comparison. J. Robot. Surg. https://doi.org/10.1007/s11701-019-01018-w (2019).

50. Mejia, A. et al. Fully Robotic Whipple vs OPEN: Early Experience. SAGES (abstract) (2015).

51. Shi, Y. et al. Short-term outcomes after robot-assisted vs open pancreaticoduodenectomy after the learning curve. JAMA Surg. https://doi.org/10.1001/jamasurg.2020.0021 (2020)

52. Wang, S. E., Shyr, B. U., Chen, S. C. \& Shyr, Y. M. Comparison between robotic and open pancreaticoduodenectomy with modified Blumgart pancreaticojejunostomy: A propensity score-matched study. Surgery 164, 1162-1167. https://doi.org/10.1016/j. surg.2018.06.031 (2018).

53. Zhou, N. X. et al. Outcomes of pancreatoduodenectomy with robotic surgery versus open surgery. Int. J. Med. Robot. 7, $131-137$. https://doi.org/10.1002/rcs.380 (2011).

54. Zimmerman, A. M., Roye, D. G. \& Charpentier, K. P. A comparison of outcomes between open, laparoscopic and robotic pancreaticoduodenectomy. HPB (Oxford) 20, 364-369. https://doi.org/10.1016/j.hpb.2017.10.008 (2018).

55. Boone, B. A. et al. Assessment of quality outcomes for robotic pancreaticoduodenectomy: Identification of the learning curve. JAMA Surg. 150, 416-422. https://doi.org/10.1001/jamasurg.2015.17 (2015).

56. Caba Molina, D., Lambreton, F. \& Arrangoiz Majul, R. Trends in robotic pancreaticoduodenectomy and distal pancreatectomy. J. Laparoendosc. Adv. Surg. Tech. A 29, 147-151. https://doi.org/10.1089/lap.2018.0421 (2019).

57. Gachabayov, M., Gogna, S., Latifi, R. \& Dong, X. D. Passive drainage to gravity and closed-suction drainage following pancreatoduodenectomy lead to similar grade B and C postoperative pancreatic fistula rates. A meta-analysis. Int. J. Surg. 67, 24-31. https ://doi.org/10.1016/j.ijsu.2019.05.001 (2019).

58. Verbeke, C. S. et al. Redefining the R1 resection in pancreatic cancer. Br. J. Surg. 93, 1232-1237. https://doi.org/10.1002/bjs.5397 (2006).

59. Esposito, I. et al. Most pancreatic cancer resections are R1 resections. Ann. Surg. Oncol. 15, 1651-1660. https://doi.org/10.1245/ s10434-008-9839-8 (2008).

60. Ricci, C. et al. Minimally invasive pancreaticoduodenectomy: What is the best "choice"? A systematic review and network metaanalysis of non-randomized comparative studies. World J. Surg. 42, 788-805. https://doi.org/10.1007/s00268-017-4180-7 (2018).

\section{Author contributions}

Conception and design of the work: X.D.D. and M.G. Data acquisition: A.R., S.G., A.A., E.Z., and M.D. Data analysis: X.D.D., E.Z., D.M.F., M.D., and M.G. Interpretation of the data: X.D.D., D.M.F., and M.G. Drafting the manuscript: X.D.D., D.M.F., and M.G. Critical revision of the manuscript: X.D.D., D.M.F., S.G., A.R., E.Z., M.D., A.A., and M.G. Final approval of the manuscript for submission: X.D.D., D.M.F., S.G., A.R., E.Z., M.D., A.A., and M.G. Agreement to be accountable for all aspects of the work: X.D.D., D.M.F., S.G., A.R., E.Z., M.D., A.A., and M.G.

\section{Competing interests}

The authors declare no competing interests.

\section{Additional information}

Supplementary Information The online version contains supplementary material available at https://doi. org/10.1038/s41598-021-83391-x.

Correspondence and requests for materials should be addressed to X.D. or M.G.

Reprints and permissions information is available at www.nature.com/reprints.

Publisher's note Springer Nature remains neutral with regard to jurisdictional claims in published maps and institutional affiliations.

(c) (1) Open Access This article is licensed under a Creative Commons Attribution 4.0 International (c) License, which permits use, sharing, adaptation, distribution and reproduction in any medium or format, as long as you give appropriate credit to the original author(s) and the source, provide a link to the Creative Commons licence, and indicate if changes were made. The images or other third party material in this article are included in the article's Creative Commons licence, unless indicated otherwise in a credit line to the material. If material is not included in the article's Creative Commons licence and your intended use is not permitted by statutory regulation or exceeds the permitted use, you will need to obtain permission directly from the copyright holder. To view a copy of this licence, visit http://creativecommons.org/licenses/by/4.0/.

(C) The Author(s) 2021 